\title{
Heaps Don't Lie: Countering Unsoundness with Heap Snapshots
}

\author{
NEVILLE GRECH, University of Athens, Greece and University of Malta, Malta \\ GEORGE FOURTOUNIS, University of Athens, Greece \\ ADRIAN FRANCALANZA, University of Malta, Malta \\ YANNIS SMARAGDAKIS, University of Athens, Greece
}

Static analyses aspire to explore all possible executions in order to achieve soundness. Yet, in practice, they fail to capture common dynamic behavior. Enhancing static analyses with dynamic information is a common pattern, with tools such as Tamiflex. Past approaches, however, miss significant portions of dynamic behavior, due to native code, unsupported features (e.g., invokedynamic or lambdas in Java), and more. We present techniques that substantially counteract the unsoundness of a static analysis, with virtually no intrusion to the analysis logic. Our approach is reified in the HeapDL toolchain and consists in taking whole-heap snapshots during program execution, that are further enriched to capture significant aspects of dynamic behavior, regardless of the causes of such behavior. The snapshots are then used as extra inputs to the static analysis. The approach exhibits both portability and significantly increased coverage. Heap information under one set of dynamic inputs allows a static analysis to cover many more behaviors under other inputs. A HeapDL-enhanced static analysis of the DaCapo benchmarks computes $99.5 \%$ (median) of the call-graph edges of unseen dynamic executions (vs. $76.9 \%$ for the Tamiflex tool).

CCS Concepts: • Software and its engineering $\rightarrow$ General programming languages;

Additional Key Words and Phrases: Program Analysis, Heap Profiling, Soundness, Instrumentation

ACM Reference Format:

Neville Grech, George Fourtounis, Adrian Francalanza, and Yannis Smaragdakis. 2017. Heaps Don't Lie: Countering Unsoundness with Heap Snapshots. Proc. ACM Program. Lang. 1, OOPSLA, Article 68 (October 2017), 27 pages. https://doi.org/10.1145/3133892

\section{INTRODUCTION}

Static analysis approaches typically attempt to be over-approximate and cover all possible program behavior: when there are two possible paths of execution, a static analysis explores both; when there are many possible values for a variable, a static analysis examines all of them, usually by employing an abstraction that groups together a large number of concrete values.

Still, practical static analyses routinely suffer from unsoundness [Livshits et al. 2015], by failing to account for standard dynamic behavior. The causes of this unsoundness are features such as

Authors'email: me@nevillegrech.com,gfour@di.uoa.gr, adrian.francalanza@um.edu.mt and yannis@smaragd.org. Authors' addresses: Neville Grech, Dept. of Informatics, University of Athens, Ilisia, Athens, 15784, Greece , University of Malta, Malta, me@nevillegrech.com; George Fourtounis, Dept. of Informatics, University of Athens, Ilisia, Athens, 15784, Greece, gfour@di.uoa.gr; Adrian Francalanza, Dept. of Computer Science, University of Malta, Msida, Malta, adrian. francalanza@um.edu.mt; Yannis Smaragdakis, Dept. of Informatics, University of Athens, Ilisia, Athens, 15784, Greece, yannis@smaragd.org.

This work is licensed under a Creative Commons Attribution 4.0 International License.

(C) 2017 Copyright held by the owner/author(s).

2475-1421/2017/10-ART68

https://doi.org/10.1145/3133892

Proceedings of the ACM on Programming Languages, Vol. 1, No. OOPSLA, Article 68. Publication date: October 2017. 
reflection, native code, dynamic loading, but also cross-language development (e.g., hybrid JavaJavascript apps or languages running on top of the JVM and integrating with the Java libraries) and the engineering complexity of supporting a growing number or more-and-more complex language features, such as Java's invokedynamic instruction. The typical modern Java application uses complex frameworks that integrate external resources (e.g., XML files) with inversion-of-control patterns that present static analysis frameworks fail to account for.

An approach for coping with the ever-increasing dynamism of realistic programs is to capture dynamic behavior and encode it as an input for subsequent static analysis. For instance, Hirzel et al. [2007, 2004] attempt to counter dynamic loading by observing its effects, recording the results, and re-running the static analysis. The Tamiflex tool [Bodden et al. 2011] records the result of reflective operations and dynamic loading actions, produces a log as an input to the static analysis, or even rewrites the program with these sources of dynamic behavior replaced by the exact behavior observed during the dynamic run.

Although these efforts have pushed the state of the art, they still fall short of capturing many sources of unsoundness, such as program semantics expressed in different languages (be it Javascript code for UI elements, or $\mathrm{C} / \mathrm{C}++$ code in native libraries) or the lack of support for cutting-edge language features (e.g., invokedynamic and lambdas). Unsound handling of such features translates into reduced analysis coverage: the static analysis misses many valid program behaviors.

Our work proposes an approach that compensates for the coverage shortcomings of static analysis by integrating dynamic information produced from heap dumps: snapshots of dynamic behavior that record the shape of the heap, the stack shape (i.e., full stack traces) when every object was created, and more. Heap dumps reflect a substantial portion of the complex dynamic behavior of a program, regardless of the cause of such behavior: instead of watching what happens at specific actions (e.g., reflection or dynamic loading operations), a heap dump records the cumulative semantic effects of program execution in its native setting and complex environment. At the same time, heap dumps do not miss the ability to capture dynamic actions (e.g., a dynamic call-graph) since each object (either natively or through heap enricher functionality that we introduce) records information describing the dynamic context at the time of its allocation.

We implemented our approach in the HeapDL tool for Java programs, on both the JVM and Android. HeapDL leverages different APIs to produce standard HPROF heap dumps, and processes them to produce representations of the heap and call graph that static analysis can use. (HeapDL also produces a packaged version of both the statically available and the dynamically loaded classes of the program.) We show the benefits of HeapDL by importing its output in standard static analyses (points-to and call-graph analysis). The result demonstrates the benefits of our approach:

- Heap dumps produce significant increases in analysis coverage, compared to past techniques that enhance a static analysis with knowledge about dynamic actions (e.g., reflection and dynamic loading). A static analysis enriched by our HeapDL tool discovers $24 \%$ more callgraph edges and $86 \%$ more references between heap objects, compared to the same analysis enriched by the Tamiflex tool. The benefit is clear in direct comparisons of the predictive power of each analysis: given the same dynamic input, the HeapDL-enhanced analysis statically computes $99.5 \%$ (median) call-graph edges of dynamic executions under different inputs, vs. $76.9 \%$ for Tamiflex.

- HeapDL heap dumps are better suited for precise integration with static analysis. Our heap enricher allows the recording of context that is otherwise not readily available. Notably, we generate context-sensitive information for object-sensitive analyses, of any context depth. This information can be directly integrated in a static analysis that uses the same context abstraction. 
- Heap dump technology is by nature more portable than dynamic agents that watch specific program actions. HeapDL supports both JVM and Android dynamic analysis, unlike past tools (e.g., Tamiflex) that are JVM-only. We argue that this is an inherent difference, rather than an outcome of current technology trends: it is more likely for a runtime environment to support snapshots of state rather than arbitrary recording of program actions during execution.

More generally, our approach follows a theme well-established in the literature: the combination of static and dynamic analysis, so that concrete information can take the place of static abstractions that are hard or impossible to compute. In this general theme, there are specific elements of our techniques that are unique, and are largely responsible for the benefits we obtain. These elements include: (a) the use of heap snapshots with state-of-the-art technology; $(b)$ the enhancing of such snapshots with extra context information and with objects that would normally not be available; (c) the packaging of dynamic information for reuse by common whole-program static analyses (such as points-to analyses or call-graph construction). We next describe our approach with an emphasis on these unique elements.

\section{OVERVIEW OF THE APPROACH}

We begin with an overview of the main elements of the HeapDL approach: the overall workflow, current heap dump technology, and output for integration with static analyses. The discussion in this section is purposely simplified. In Section 3 we discuss how we enhance the basic scheme.

\subsection{Motivation and Main Idea}

The HeapDL approach consists of taking snapshots of a running program's heap and using them to provide further input for a static analysis. The intent is to uniformly capture the state-changing effects of hard-to-analyze features. These features include native and other heterogeneous code, cutting-edge language features, dynamic loading, and more. A modern application crucially depends on such features, yet static analysis frameworks (such as the Soot infrastructure [Vallée-Rai et al. 1999] or the Doop pointer analysis framework [Bravenboer and Smaragdakis 2009]) have incomplete support for them. Examples in the Java world include:

- Virtually all modern Java programs have semantics that depend on native code. For instance, atomic operations are essential for high-performance shared-memory parallelism. Atomic reads and writes on the heap (e.g., to object fields or array entries) are implemented as native Java methods. If the static analysis does not model all of them, it will miss significant state updates. It is as essential for an analysis to model, e.g., native method sun.misc. Unsafe. compareAndSwapObject as it is to support plain heap load and store instructions. Yet doing so is hard. Extra native operations get added in every release of the JDK and analysis authors typically do not keep up with them. These operations can be much more numerous than JVM instructions. On a quick count, there are over 6,000 native methods in OpenJDK 8 u60 (vs. under 200 instruction opcodes in the JVM instruction set).

- Most enterprise or mobile Java programs heavily leverage complex frameworks, effectively becoming heterogeneous applications. For example, an Android app is a complex composition of UI elements, whose specification is in XML, and Java code. Upon loading, the XML specification is used to instantiate many graphical components, which can also be referred to from plain Java code via dynamic lookups (using integer keys). Similar examples of framework usage abound-in Servlet coding, J2EE applications and more. Java Enterprise frameworks heavily employ XML specifications, with inversion-of-control patterns used to determine how plain Java code is invoked. Static analyses attempt to capture the semantics of such frameworks to the extent possible. E.g., the FlowDroid [Arzt et al. 2014] add-on to the Soot 


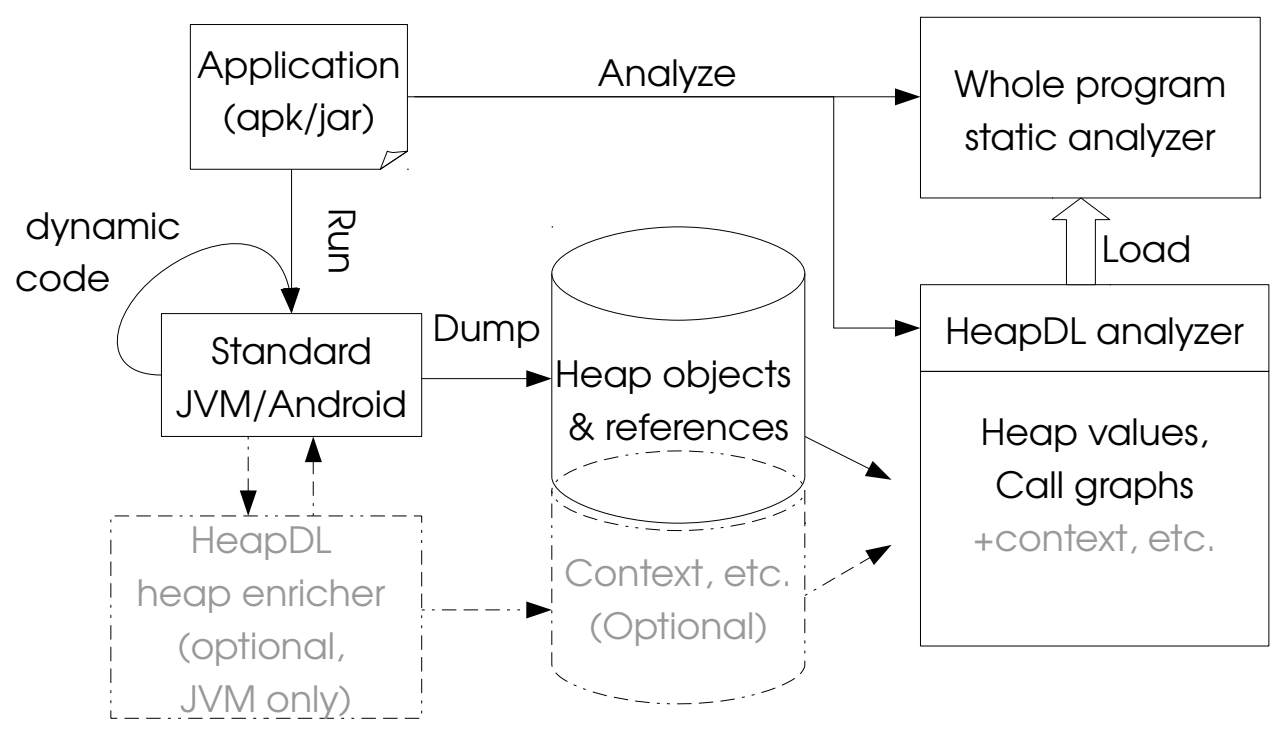

Fig. 1. Design of HeapDL

framework implements basic processing of Android XML layout files. Yet such support is always vastly incomplete (as will also be apparent in our experimental evaluation) due to the complexity and ever-changing nature of modern frameworks.

- Even with the limited size of the JVM instruction set, static analyses do not fully support it. Java 7 introduced a new bytecode opcode, invokedynamic [Rose 2009], together with an API (for "method handles") around it, that can offer the programmer the capability to completely customize dynamic program behavior. The invokedynamic functionality is used to implement dynamic languages on the JVM and also a growing number of dynamic features of Java (e.g., lambdas [Oracle 2014b], string concatenation [Oracle 2017], or generics specialization[Goetz 2016]). To this date, support for invokedynamic in static analysis frameworks has been, at best, incomplete.

All the above instances result in unsoundness; the static analysis fails to capture actual dynamic behavior. This unsoundness is quantified as reduced coverage of program behavior. HeapDL compensates by adding dynamic information to static analysis. Semantic effects, captured by the heap state and dynamic call-graph of the application, are extracted from a heap dump and used to supplement a static analysis. Figure 1 shows the main components, schematically. HeapDL relies on profiling capabilities of the target runtime. Both major Java-based platforms, Android and the JVM, provide multiple memory profiling and heap dumping solutions. With an enriching agent (Section 3) we can make a heap dump encode even more information that is of direct value to static analysis.

The dynamic information is output in a form suitable to import in a static analysis. HeapDL explicitly targets whole-program analyses, rather than local static analyses. It is, for instance, much better suited for points-to analysis, heap shape analysis, or call-graph construction, rather 
than for symbolic execution or model checking. This is reflected both in the choice of technology for capturing dynamic information (Section 2.2) and in the packaging of information for reuse (Section 2.3).

To see how a heap snapshot can counter the effects of unsoundness in static analysis, we can consider some concrete examples.

Example: external code effects. Consider an Android application, with several Java components linked together by means of an XML specification. By taking a snapshot of the running application, HeapDL can capture behaviors that are very hard to follow via static analysis alone. For instance, the instantiation of UI components and their inter-linking (e.g., a window object contains a reference to three panels and a slider) will be hard to detect statically, since it is implemented deep in the Android runtime, in large part in native code. A heap snapshot can inform the static analysis about the instances of these UI components and their inter-connectivity. In this way, the analysis starts from a valid initial setup and can cover substantially more code (e.g., by statically analyzing possible called methods on these components).

Example: better reflection analysis. Even if we focus only on reflection analysis, heap snapshots can offer advantages compared to merely recording dynamic reflective actions. Consider a program that holds a large array of $k \approx 1000$ class names, initialized so that no static analysis can know its values (e.g., read from an XML file). These class names can represent different to-be-loaded components (e.g., plug-ins of a large application). The class names can be used to call methods via reflection. In a single execution, a small number, e.g., 3, distinct class names are used. Current state-of-the-art tools for handling reflection, such as Tamiflex, watch dynamic reflective actions and hence record the calls to the 3 classes' methods. Therefore, a static analysis enhanced with Tamiflex output can also analyze the 3 reflective calls. HeapDL takes a heap snapshot, so it can capture all $k$ members of the array. A static analysis enhanced with HeapDL output and with minimal reflection logic will analyze all possible calls to all $k$ classes' methods.

Example: handling extra language features. Consider a static analysis that does not handle the invokedynamic instruction or its associated method-handles API. Heap snapshots can alleviate the effects of such unsoundness in two ways. First, a heap snapshot also includes snapshots of dynamic call graphs, and can, therefore, capture the target of an invokedynamic call. Second, the heap effects of the method called via invokedynamic are captured in the snapshot. In this way, a static analysis enhanced with HeapDL input can attain significantly higher coverage of program behaviors that employ invokedynamic calls.

Generally, heap snapshots can capture complex dynamic behavior that is otherwise invisible to a static analysis and augment the static analysis with such information.

\subsection{Background: Heap Dumps, Allocation Tracking}

HeapDL implements a heap dump analyzer that accepts standard HPROF [Oracle 2016a] heap dumps. Java or Android applications are dynamically executed by running on the unmodified, standard Java Virtual Machine or Android runtime.

A heap dump is primarily a complete encoding of a program's heap as a graph, i.e., a snapshot of all interconnections between heap objects. Heap dumps can contain anything that is loaded or computed by the application or VM, i.e., not just any normal heap objects constructed by the application but also primitives, class objects, and strings. This view, however, is too poor to capture the wealth of information available through heap-dump APIs. In our setting, when we refer to a heap dump, we mean a heap dump with allocation tracking: each heap object records a full stack 
trace of the run-time context at the exact allocation instruction. Allocation tracking has a runtime cost but is a portable facility, uniformly available in modern heap dump APIs. By leveraging allocation tracking, a typical heap snapshot also integrates many thousands of stack snapshots, at earlier points of the execution (i.e., whenever a heap object was allocated). These stack snapshots are significantly condensed, containing merely call-graph edges (i.e., which instruction called which method) rather than full stack contents. (In Section 3 we see how we force the collection of even more stack information, via our enriching agent.) This is, however, highly valuable information for enhancing the coverage of a static analysis. Compensating for the unsoundness of reflection, dynamic loading, invokedynamic, inversion-of-control patterns, etc. is majorly facilitated by these dynamic call-graph snapshots.

\subsection{Output Schema}

HeapDL accepts as input both the program code and a heap dump. It then distills the heap dump into input tables for a static analysis, by mapping objects and call-graph elements to abstractions. These abstractions are derived by consulting the program code. HeapDL then outputs the tables in standard text form, as comma-separated value files, with appropriately externalized identifiers.

In Figure 2, we present a schema of the domain of tables created by HeapDL for consumption by a context-insensitive static analyzer. The heap relations generated by HeapDL bridge the gap between the domain of an application's state and the domain of static analysis. The relation OBJECTFIELDVALUE captures what values an object's fields can point to, and similar information is kept for static fields of a class (StaticFieldValue) and arrays (ArrayContentsValue). CallGRAPHEDGE captures the dynamic call-graph: every pair of successive stack trace elements forms an edge. That is, the call-graph is the union of all call-graph edges taken from the (large number of) stack snapshots collected due to allocation tracking. Notably, we have found that call graphs constructed in this way are comparable in size and information content with ones created using explicit instrumentation of calls. (This is perhaps not too surprising: object allocation is frequent and virtually all meaningful call chains will reach code that causes at least one allocation, possibly of a temporary object, resulting in the call chain's capture.) Instrumentation, through Java or native agents, is a less portable technique than heap snapshots, however-e.g., there is no Android API for user-defined agents; bytecode rewriting can be used but fails for native code or system classes.

All of the above mappings employ different kinds of abstraction: objects are mapped to abstract objects, array contents are merged, the union of field-points-to sets (per abstract object) or callgraph-edge sets (per invocation site) is taken. Our heap object abstractions, $O$, match those typically used by whole-program static analysis frameworks, i.e., usually represent allocations sites:

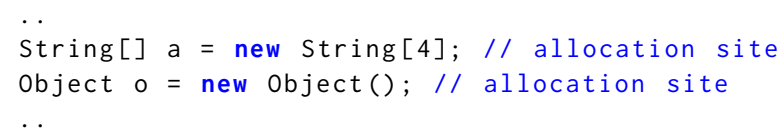

On the other hand, when statically modeling string constants and class objects, the identity of these is used as the object abstraction, instead of their allocation site. For instance, the identity of classes is the fully qualified name (and the classloader if the static analyzer can distincguish classes with the same name loaded by different classloaders). The identity of strings can also be their content if the static analyzer is tracking strings for the purpose of static reflection analysis.

To generate dynamically inferred heap relations, HeapDL must first find the right object abstractions from the heap dump. This is often a best-effort match. HeapDL walks the allocation traces and uses heuristics to find the most probable frame where the real allocation site is found as a first 


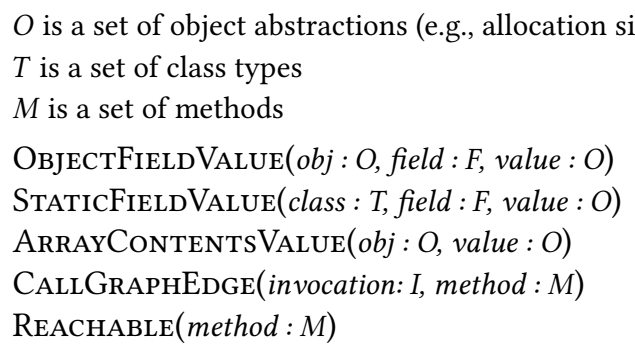

$F$ is a set of fields

$I$ is a set of instructions

Fig. 2. Our domain, for context-insensitive heap relations extracted by HeapDL

approximation. Given this approximation, it tries to then match by type, line number, and other information. Some of this information is only present in debug information of the bytecode. Since the line number is not always guaranteed to be present in the application under analysis, matching is sometimes done just by method descriptor and type. In cases were the actual code is not statically available, a dummy abstract object allocation site containing the right type information is generated. This typically happens due to either native code, foreign code, or cutting-edge language features such as lambda meta-factories that generate transient classes and are incompletely modeled.

With the above schema, the information that HeapDL provides to static analysis is compact and in line with current static (whole-program) points-to analyses or call-graph construction. A static analysis typically only needs to import the HeapDL information and consider it as ground facts, before it starts its own further propagation of values. In general, the integration of HeapDL into an analysis toolchain is similar to that of past tools, such as Tamiflex: dynamic execution yields call-graph edges and object references, in an externalized format (comma-separated value files). An analysis-specific import method subsequently performs a straightforward mapping from the externalized information to the structures that the analysis uses to represent its own inferences.

Since static analysis is fundamentally over-approximate, small amounts of provided information (e.g., a few hundred extra call-graph edges or values in object fields that were previously undetected) are often responsible for making the static analysis compute a much larger number of inferences.

\section{ENRICHING HEAPS AND CONTEXT SENSITIVITY}

Enriching heaps is a process of strategically making small additions to the state of the application so that a heap dump maintains more information that we would like to produce as input to a static analysis. This technique leverages the state-preserving abilities of the profiling toolchain. It also preserves the actual linking between objects and references of the original state of the application with that of the additional information.

There are three main ways that the HeapDL context enricher injects additional information into the state of the application:

- Adding new references within agent code, e.g., during class loading.

- Injecting code into the application to add new references within the application.

- Injecting code into the application to create new objects at strategic program points.

These additions are typically made using instrumentation agents, in Java or in native code, through standard APIs of the JVM. HeapDL currently only supports heap enriching on the JVM platform, since Android does not have a standard API for agents.

HeapDL performs heap enrichment for several different purposes, detailed next. 


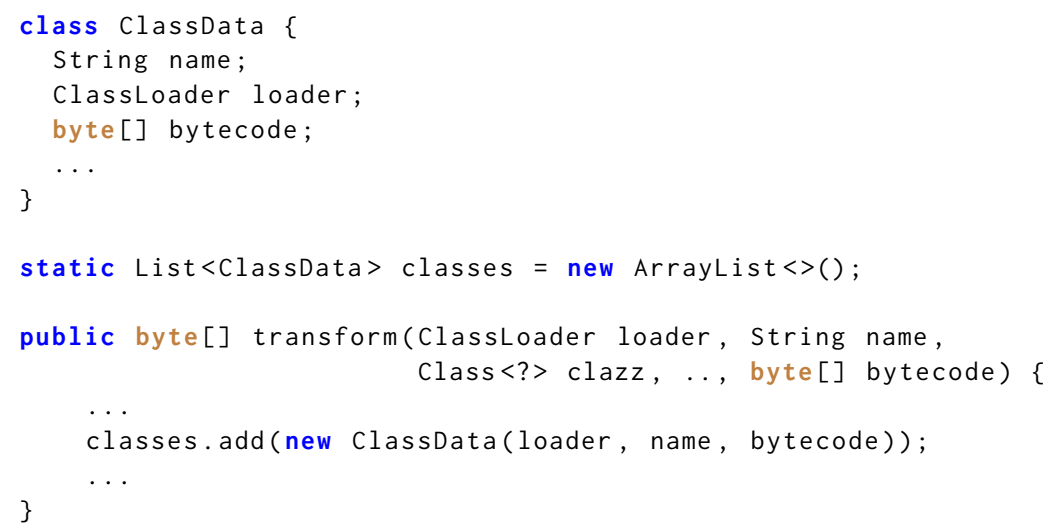

Fig. 3. Heap enricher example: Enriching heap with bytecode of loaded classes (code).

\subsection{Capturing Dynamically Loaded Code}

HeapDL captures all dynamically-loaded bytecode and packages it for use by a static analysis. This is beneficial, since dynamically-loaded classes (including temporary dynamically-generated code-e.g., for invokedynamic and other method-handle API support) would not otherwise be available for static analysis. This general pattern has also been present in past work. For instance, Tamiflex [Bodden et al. 2011] creates an archive file with loaded classes by instrumenting class-load events via an agent.

The complication, however, is that a loaded class is not uniquely identified by its name (or its bytecode, as provided to the loader). In a running JVM, a class's identity is represented by a combination of its static identity (i.e., its name, which is an artificial id for internally-generated, anonymous classes) and its class loader object (an instance of type ClassLoader) [Lindholm et al. 2014, §5.3]. The class loader can arbitrarily transform the loaded bytecode. Therefore, an approach that records bytecode by capturing the inputs of class-load events (before actual loading has taken place) is incomplete: the uniquely-identifying version of a class is only available after loading is complete.

Enriched heap dumps can solve this problem and capture loaded classes together with their instances on the heap. In a plain, un-enriched, heap dump, objects do not refer to their classes' bytecode, as this is compiled away by the VM. Instead, we can instrument the code (at the point of loading classes) to perform a simple addition to the state so that dynamically loaded bytecode is captured. Figure 3 shows the skeleton-we omit features such as error handling, logging, performance optimizations, etc. for clarity.

We can see in Figure 3 that capturing the loaded bytecode can be achieved by storing, on line 13, a reference to the ClassLoader, the fully qualified name of the class, and the bytecode used to create it. With this technique there is no need for extra logic to package the classes. We use this combination of objects as keys and the structure of the heap dump contains links from class object references to their bytecode via this key. Additionally, the heap dump contains links from each instance object to its class object, and with the unique name-loader combination we have the set of associations shown in Figure 4: from every object, we can get its (dynamic) class and bytecode. 


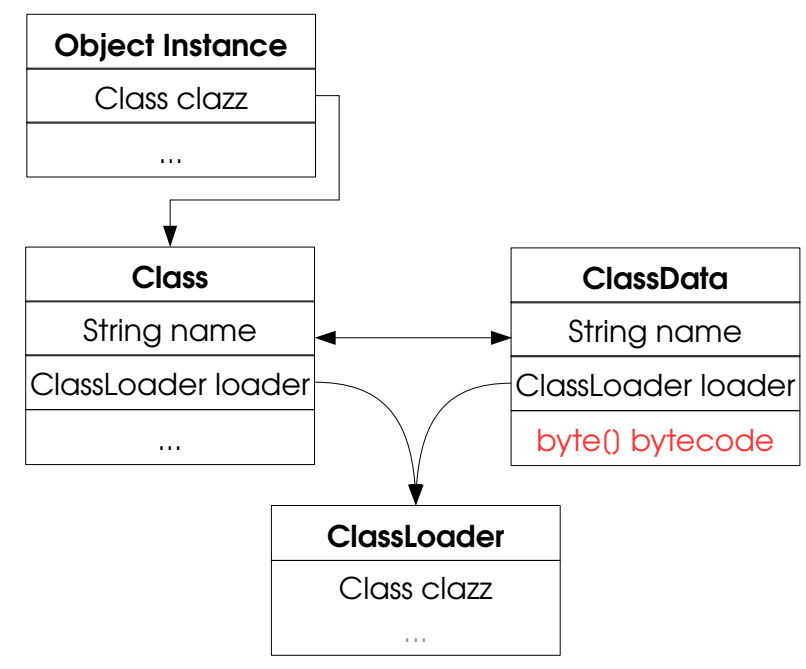

Fig. 4. Heap enricher example: Enriching heap with bytecode of loaded classes.

\subsection{Context Sensitivity}

Whole-program static analyses often employ context sensitivity to increase precision. Context sensitivity consists of qualifying all analysis inferences with special "context" entities, so that different dynamic executions are distinguished. The two main kinds of context sensitivity are call-site sensitivity [Sharir and Pnueli 1981] and object sensitivity [Milanova et al. 2005], with several alternatives and mixes proposed (e.g., type sensitivity [Smaragdakis et al. 2011] and hybrid object-call-site sensitivity [Kastrinis and Smaragdakis 2013]).

It is, therefore, desirable to provide context-sensitivity as an option for HeapDL heap snapshots. Since HeapDL has full access to dynamic information, it makes sense to preserve at least as much precision as the static analysis seeks to achieve. This requires a) creating a general infrastructure to instantiate and manipulate arbitrary context; b) capturing context not usually present in heap dumps.

Heap dumps with allocation tracking require no extra effort to support call-site sensitive contexts. In call-site sensitivity, context consists of a tuple of call sites (i.e., invocation instructions) that identify "callers". For a call-graph edge, the context of the called method is its caller, the caller's caller, and so on, up to a maximum context depth. Similarly, an allocated object's context is the caller of the method that allocated it, the caller's caller, etc. This information is naturally present in dynamic stack traces, which yield information for relation CALLGRAPHEDGE and for every allocated object on the dynamic heap (via allocation tracking).

In contrast, object sensitivity is not possible to implement from stack traces alone-our enriching agent has to maintain extra information. Object-sensitive context is a tuple of abstract objects, representing the receiver object of different calls. For a call-graph edge, the context of the called method is its receiver (abstract) object, rec; the receiver, rec2, of the method call that allocated rec; the receiver of the method call that allocated $r e c 2$; and so on. Similarly, an allocated object's context is the receiver of the method call that allocated it, the receiver of the call that allocated the former receiver, etc. 


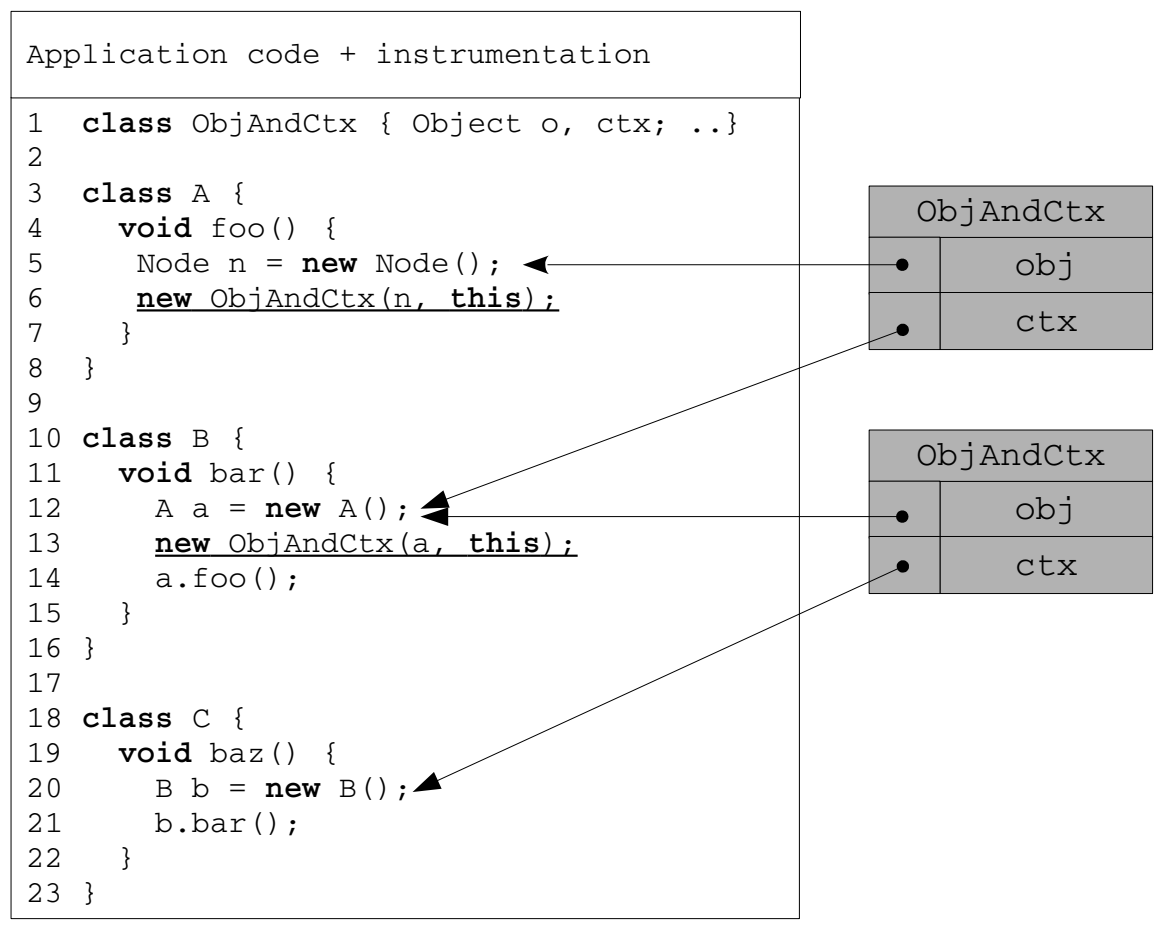

Fig. 5. Enriching the state with object sensitive heap contexts

Object sensitivity is both valuable in practical analyses and an excellent example of our heap enriching mechanisms for context sensitivity. We describe its support next, on the two key parts of heap dump information: context for heap objects and context for methods in a dynamic call-graph.

3.2.1 Storing Heap Contexts on Object Creation. In order to support object sensitivity, HeapDL maintains extra context information per allocated object. This is done via a class ObjAndCtx that associates each dynamic object with its allocation context. HeapDL instruments the application code to allocate instances of ObjAndCtx every time a regular object would be allocated. The HeapDL heap enricher is implemented as a Java agent that performs load-time structured bytecode transformations. This is by no means the only way to implement such a strategy. Other ways include native agents, aspect oriented programming with bytecode weaving, and more.

Figure 5 shows a target program with additional instrumentation for object sensitivity. The tuple structure that represents the heap context of an object appears at Line 1 . This is injected into the classpath of the application and instances of this structure are created on lines 6 and 13. At any interesting program points where an object is constructed, the instrumentation keeps track of the receiver of the current method. 


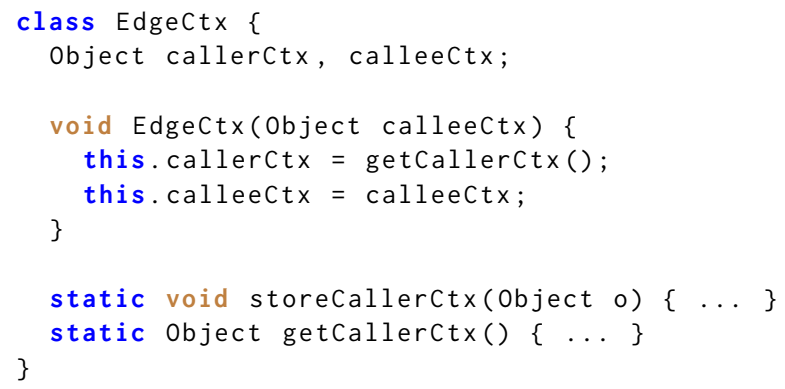

Fig. 6. Edge Context

Note that, even though one receiver object is kept, object-sensitive context of any depth can be computed: the receiver object contains a reference to the receiver object of its own allocation method, etc.

The example shows objects constructed within instance methods. Objects created inside static methods are handled differently, since these have no receiver object, so the receiver of the caller is used for the purposes of heap contexts. Every currently active stack frame also keeps track of its receiver object, not shown in the figure.

HeapDL keeps track of context information only inside application code, to focus on the cases that require maximum precision, and to avoid errors with the instrumentation of libraries. It also economizes by not tracking the context of commonplace objects (which is typically not statically modeled), such as primitive arrays, strings and string buffers.

3.2.2 Storing Calling Contexts for Context-Sensitive Call Graphs. The second piece of information output by HeapDL that needs to be context-qualified is call-graph edges. Figure 6, shows an additional simple data structure, EdgeCtx, inserted into the application's class path and storing the calling context of a call-graph edge. We can see that each EdgeCtx object contains a reference to the caller context and the callee context. The storeCallerctx method is used by the instrumentation to keep track of the receiver of the caller, which is then used during the creation of the object by getCallerctx.

The enriching agent adds code to allocate a new EdgeCtx at every method call. When a new EdgeCtx object is instantiated, a stack trace is created, as illustrated in Figure 7. In the stack trace, HeapDL can extract the call-graph edge's source and target from the 2nd and 3rd elements. In the case of object sensitivity, as in our earlier discussion, the explicit context pointer is to a single object, however one can extract context of any depth by following the pointers to the (context) objects and retrieving their own allocation context.

An interesting observation is that Edgectx objects are by themselves a representation of dynamic call-graph edges, and, indeed, the most precise one. Without Edgectx objects, a heap dump has no representation of a specific dynamic call-graph edge, only of its mapping to source and target pairs, as found in a stack trace (kept via allocation tracking). Unlike the earlier ObjAndCtx structures, which give context to dynamic objects (which exist in the heap dump), Edgectx instances cannot be uniquely mapped to other heap dump entities.

3.2.3 Producing Context Sensitive Information for Consumption by Static Analyzers. Figure 8 contains a refined version of the domain and output relations extracted by HeapDL in Figure 2. In order to show how our output relations are built we need to further distinguish between abstract 


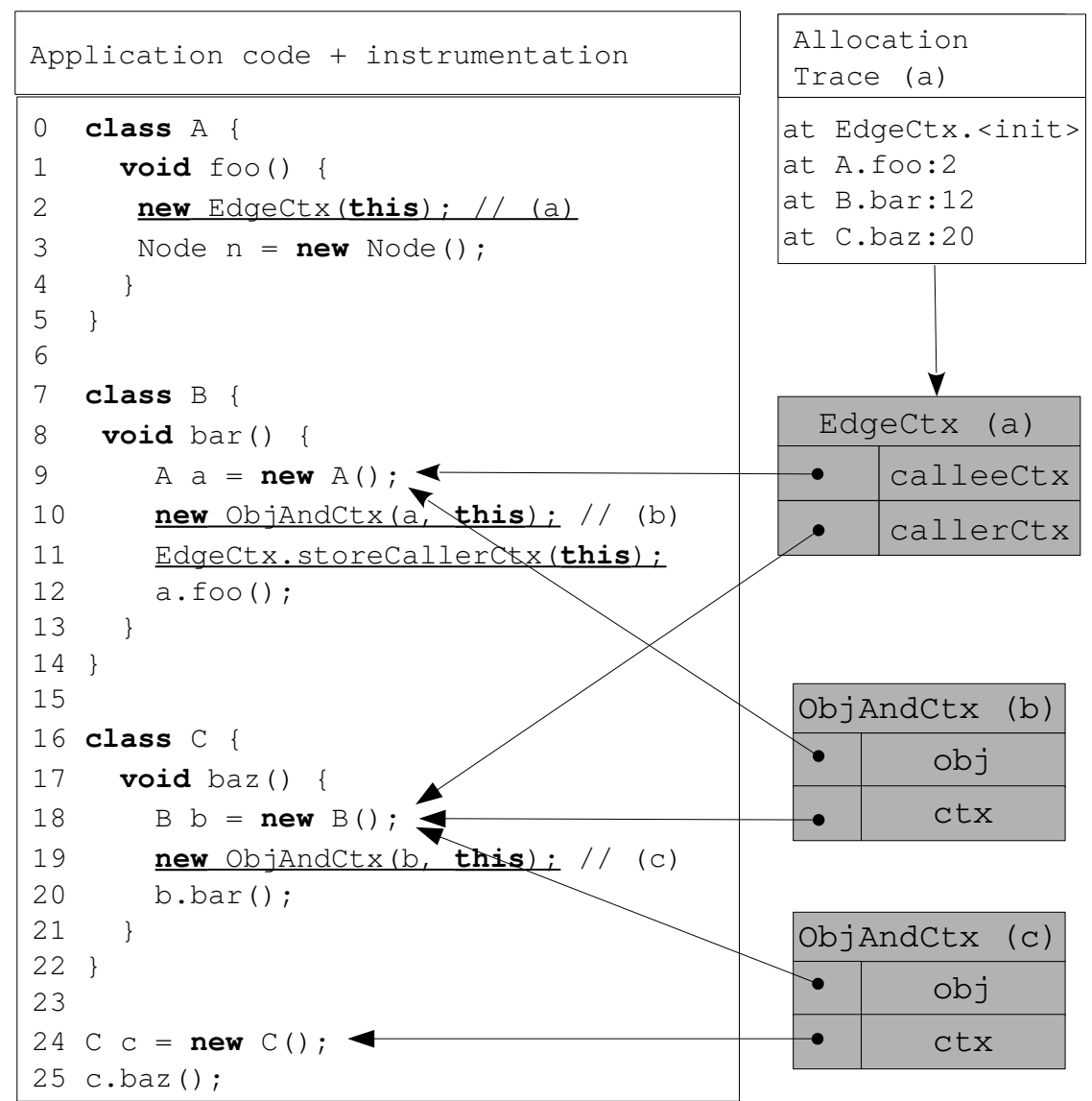

Fig. 7. Original application code, instrumentation by context heap enricher (underlined), and the depiction of the interaction between instrumentation heap objects (gray), allocation traces, and original heap objects.

objects, $O$, i.e., the objects used in the domain of static program analyzers, and concrete objects, $L$. These concrete objects are ones that are found inside the heap dump, including allocation-trace objects. Context-sensitive static analyses have parametric order in their contexts, and the calling and heap context orders can be different. (An example of this is a 2-object sensitive analysis with a 1-object sensitive heap context.) In our domain, $n$ and $m$ are the orders of the calling and heap context respectively.

In heap dumps analyzed by HeapDL, concrete contexts $L_{C}$ are a subset of concrete objects $L$. Note how, in earlier examples (Figures 3 and 6), all kinds of dynamic context shown were of type object. This is done on purpose: dynamic information maintains full detail until the moment it is packaged into output relations. Concrete contexts are then abstracted to abstract context components, $C$. The process differs, depending on the kind of context sensitivity that is employed. This process is defined using an abstraction function $\alpha$. For example, in the case of object sensitivity, the abstraction function used is the same as the abstraction function used to map any concrete object in $L$ to an 
lot more dynamic behaviors than those actually observed during the profiled program run. This is a fundamental feature of the approach: it captures not-seen behaviors and, as expected in an over-approximating static analysis, some of them may be spurious.

For a toy example, consider a method that performs a virtual call over a value read from the heap:

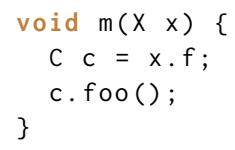

An actual program run may observe a call to method $m$ while $x$. $f$ holds an object of type $\mathrm{C} 1$ (a subtype of C). An approach, such as that of Tamiflex, that watches dynamic events will record the call-graph edge from $m$ to $\mathrm{C} 1$. foo(). However, the field $x$. $f$ may hold several different values during the program's execution. Furthermore, the over-approximating nature of static analysis may infer that $x$. $f$ points to several different values during program execution-e.g., even if each concrete object only holds a unique value in its $f$ field, grouping concrete objects into abstract ones can yield multiple values for $x . f$.

Consider a case where, later in the program's execution, $x . f$ acquires a second value: an instance of a class C2, also a subtype of C. If a HeapDL snapshot captures that value, the static analysis will consider it and will yield a call-graph edge from $\mathrm{m}$ to $\mathrm{C} 2$. foo(). This does not correspond to any observed program execution and may even be spurious: there is no guarantee that method foo is ever invoked on that value of x.f.

Therefore, HeapDL can increase the analysis reach: it models more program behaviors than approaches that only observe dynamic events. At the same time, it is interesting to evaluate whether the increase in reach is reasonable (and not the result of vast imprecision) as well as whether it corresponds to an increase in coverage of actual program behaviors.

\section{EXPERIMENTAL EVALUATION}

In this section we present the results of an experimental evaluation of HeapDL. This evaluation intends to answer the following research questions:

RQ.A Is HeapDL effective? (1) Does it expose new information that is not currently picked up through static analysis? (2) What impact does this have on the results of the analysis? (3) Does this gain also occur when explicit support for reflection is switched on in pointer analysis? (4) Furthermore, does HeapDL find additional information that a state-of-the-art runtime analysis system like Tamiflex does not?

RQ.B Is HeapDL efficient? (1) What is the additional dynamic analysis burden (i.e., at program run time)? (2) How much does the additional information add to the static analysis time?

RQ.C Does HeapDL increase coverage of the analysis, compared to a state-of-the-art runtime analysis tool?

The size of the call graph, measured by the number of call graph edges, is used as metric in $R Q . A$ and RQ.B. In addition, we use the heap size as a metric in RQ.A. The heap size is the cumulative size of relations, ArrayValue, InstanceFieldValue, and StaticFieldValue, as described in Figure 8.

The HeapDL analyzer is implemented ${ }^{1}$ as a plain Java application that produces tables in commaseparated-values format. We used the Doop framework [Bravenboer and Smaragdakis 2009] as a static analysis that accepts HeapDL input. HeapDL has integrations with two different implementations of Doop, that use the Soufflé [Jordan et al. 2016] and LogicBlox [Aref et al. 2015]

\footnotetext{
${ }^{1}$ Available online at https://github.com/plast-lab/HeapDL and http://heapdl.nevillegrech.com
} 
Datalog dialects, respectively. The integration with Doop so it can import HeapDL information is minor, consisting of merely importing data and considering them analysis facts. Doop has full support for complex Java language features, such as class initialization, exceptions, reflection, etc. In addition, Doop has recently acquired state-of-the-art support for Android applications [Grech and Smaragdakis 2017]. It specifically models the Android lifecycle, callbacks, GUI components, etc. Hence, enhancing Doop's coverage is not trivial. To parse heap snapshots, HeapDL uses a modified version of JHat [Oracle 2016b], the reference Java heap analysis tool supplied as part of OpenJDK. The modifications consist of error recovery and the addition of class pool information. For bytecode engineering, HeapDL's context heap enricher uses the ASM framework [Bruneton et al. 2002]-popular for tools that manipulate or analyze Java bytecode.

Our runtimes are established on an idle machine with an Intel Xeon E5-2687W v4 3.00GHz with up to 512 GB of RAM. For static analysis with Doop, we used the PA-datalog engine, a publicly available, stripped-down version of the commercial LogicBlox Datalog engine. We proceed in the next sections with the experiments using popular Android applications, and JVM experiments on the DaCapo 9.12-Bach benchmark suite [Blackburn et al. 2006].

\subsection{Android}

The first experiment compares the results of static analysis enhanced with HeapDL output vs. plain, unenhanced context-insensitive static analysis. We test a diverse set of Android benchmarks, chosen to be realistic applications: Chrome, Instagram, S Photo Editor, Pinterest, Google Translate, and Android Terminal Emulator.

We use Android 7.1, since it has support for heap profiling using the same HPROF format as OpenJDK. We recompiled Android from sources and produced two artifacts: (a) a JAR containing all bytecode corresponding to the Android Java API and (b) an accompanying Android virtual device image that can be loaded to the Android emulator. The reason that we generated a custom JAR for the Android API is that the JAR files that come with the Android SDK are stubs, i.e. they only contain entry points to the API methods, plus some minimal bytecode. This "full" JAR was given to the Doop static analysis as the platform JAR and permits the analysis of the app on top of Android.

To dynamically exercise the applications, we ran our benchmarks in the Android emulator with the UI/Application Exerciser Monkey tool, ${ }^{2}$ which generates random input events, to simulate actual use of each app. We performed at least 1024 random events. For some applications, we conducted two runs: one to supply log-in credentials manually, and another (after application shutdown and restart) to run with Monkey. The heap dump was taken at the exact point Monkey sent the last event. The statistical variability in Android is very low and is eclipsed by other factors such as the number of random events and user input.

Compared to later experiments, there are a few features of the Android experiment to remember: (1) the baseline is a static analysis with static support for Android features but with no dynamic information-later experiments will compare with the Tamiflex tool, which currently is JVM-only; (2) there is no heap enrichment on the Android platform.

The impact of HeapDL on the static analysis results (call-graph edges and heap size) is shown in Figures 9 and 10. Every benchmark is run in four configurations: no heap information or reflection support (base), HeapDL information (+heap), static reflection support (refl), and both reflection support and HeapDL information (refl+heap). Reflection support is the "classic-reflection" mode of Doop, which analyzes reflective features and reasons about strings used for reflection purposes.

As can be seen, the increase in all metrics due to the use of heap snapshots is drastic. Many tens of percent of extra call-graph edges and an equal or larger increase in static heap size occur. The

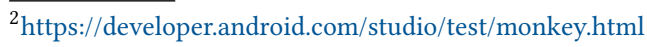




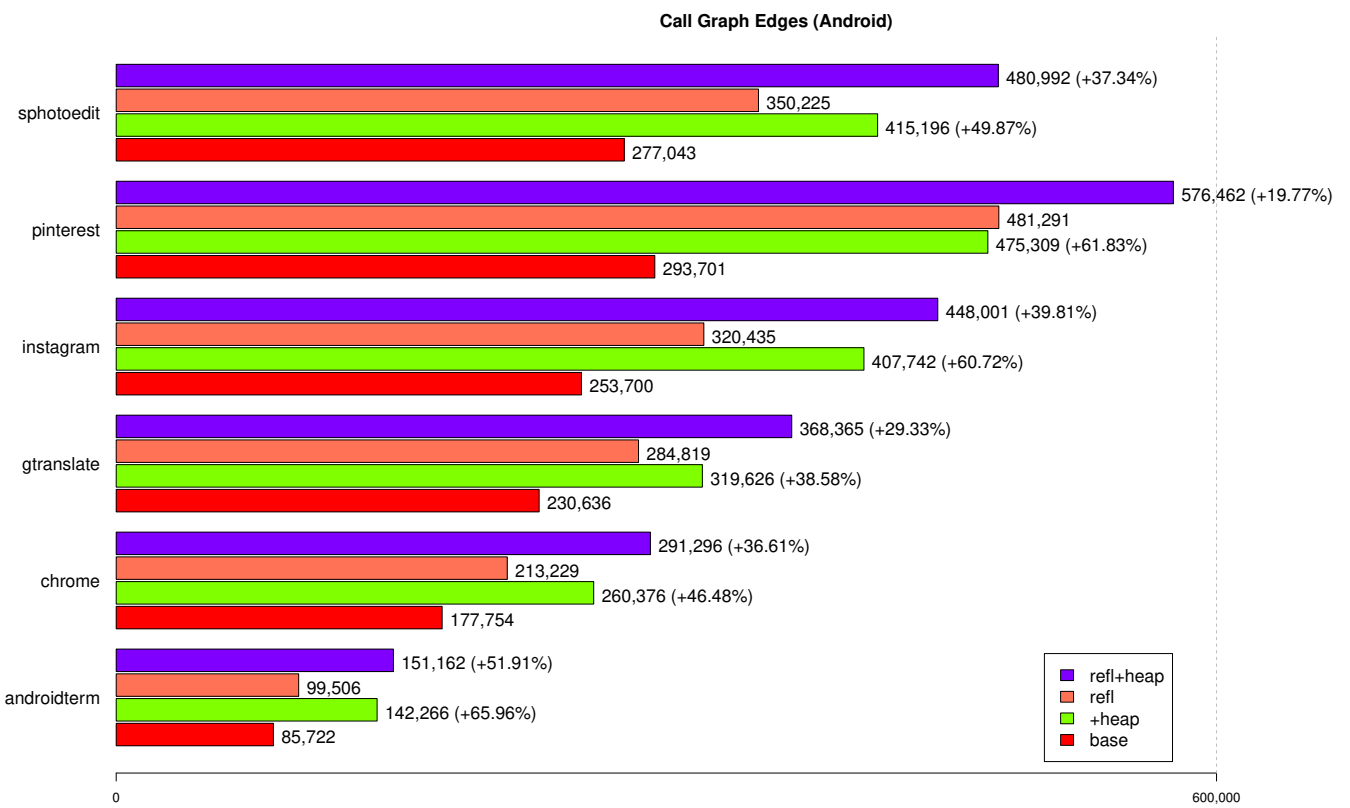

Fig. 9. Android benchmarks: number of call-graph edges reported by the static analysis, with and without HeapDL assistance, with and without static reflection analysis.

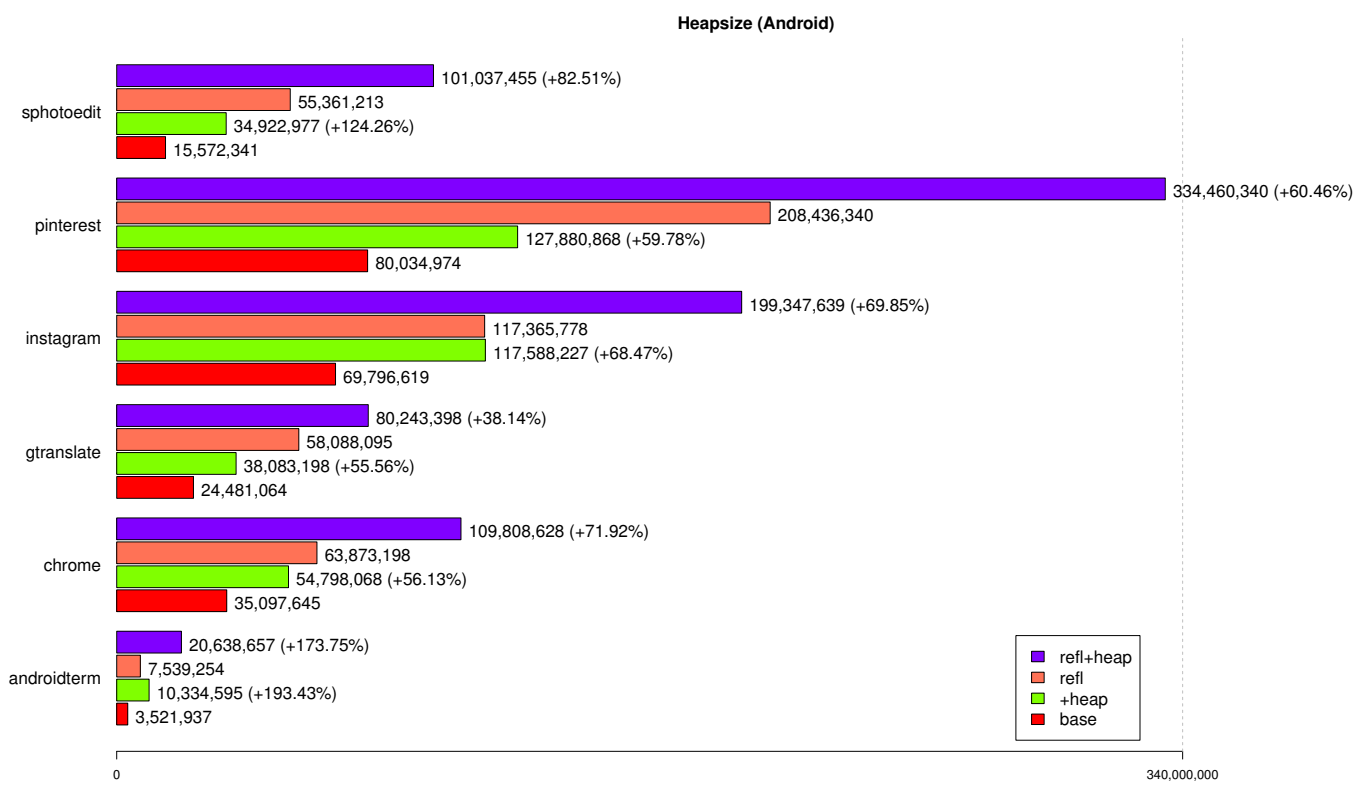

Fig. 10. Android benchmarks: heap size reported by the static analysis, with and without HeapDL assistance, with and without static reflection analysis. 


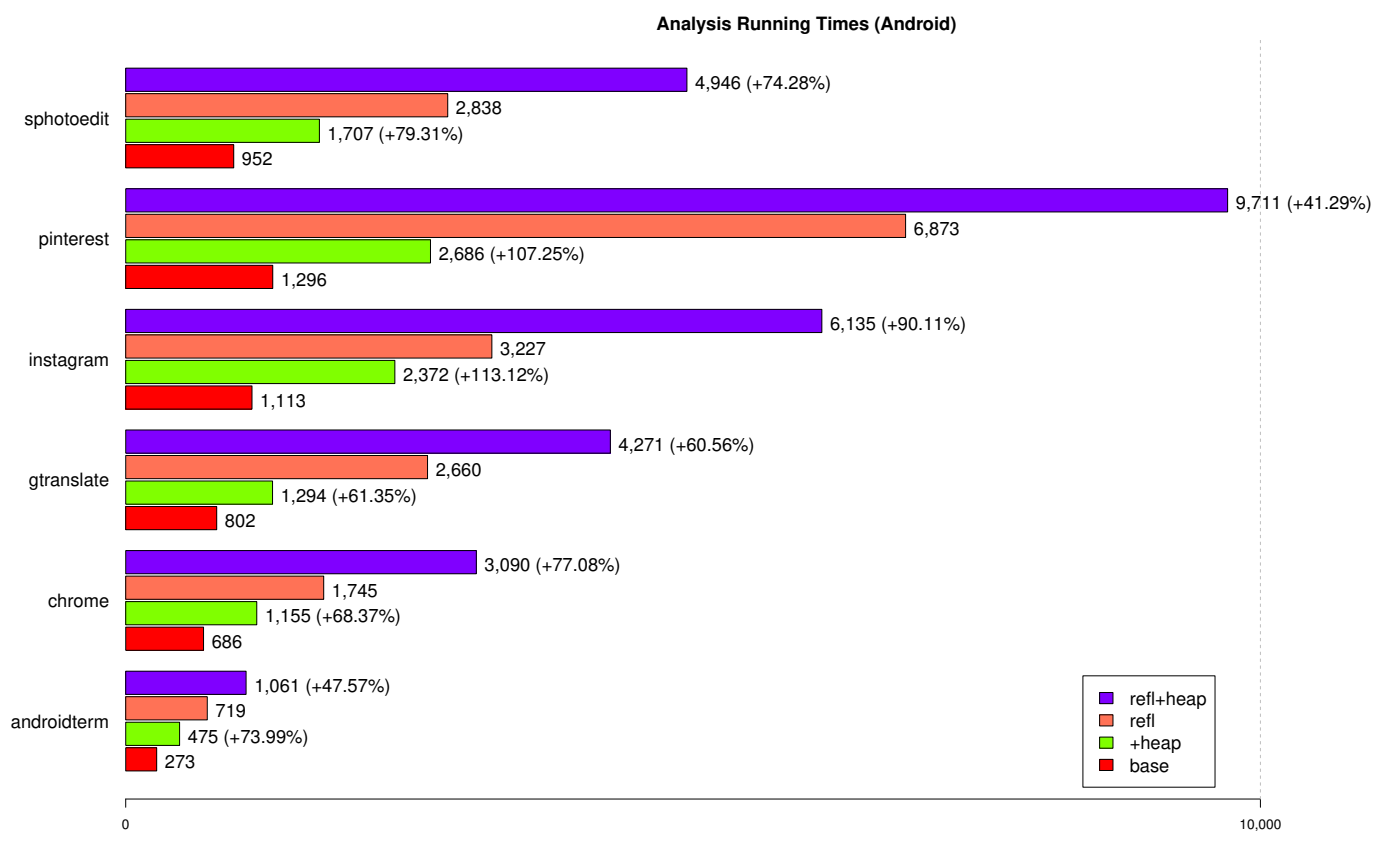

Fig. 11. Analysis times for Android benchmarks.

static analysis reach expands significantly-the static analysis on its own is not enough to discover this extra information, strongly suggesting unsoundness. Even with static reflection analysis, the increase with HeapDL input is large. All metrics support the position that HeapDL is effective (RQ.A1-3). (The Android platform does not easily permit dynamic instrumentation to see if the extra static analysis results really capture valid dynamic behaviors-our later experiments will address this.)

Figure 11 shows the running time of the static analysis when enhanced with HeapDL input. The running time increase (typically in the $60-80 \%$ range) is commensurate with the increase in overall analysis reach. Running times remain realistic, considering how much more code is analyzed (as evident by the extra call-graph edges) and the overall size of the programs involved-some of the largest Android apps are included in our benchmark set. This suggests that HeapDL is efficient and does not burden the static analysis disproportionately (RQ.B2).

Regarding the cost of the dynamic analysis (RQ.B1), interestingly, we did not find a significant overhead when allocation tracking is turned on in Android. Measuring application start-up, runtime, shutdown times, and their sum, with and without allocation tracking, we observed a significant variance (up to \% 10) between runs of the same benchmark, but without any strong correlation with allocation tracking being enabled. This could be due to the interactive nature of most of the tested applications, ${ }^{3}$ or to other overheads of the system. We investigated this topic further by hand-crafting an Android application that performs no I/O. Under this synthetic scenario we observed a worst-case $48 \%$ overhead of heap snapshots with allocation tracking. It is telling that we had to resort to a synthetic benchmark to obtain a measurable overhead.

\footnotetext{
${ }^{3}$ We had to set a delay of $2 \mathrm{~ms}$ in the Monkey tool to avoid losing events. If a mere $2 \mathrm{~ms}$ interaction time is sufficient to hide profiling slowdown, it can be well argued that there is no perceptible slowdown in the first place.
} 


\subsection{JVM Benchmarks: DaCapo}

Our second experiment examines the standard DaCapo 2009 Java benchmark suite on the JVM. ${ }^{4}$ We omitted a priori the Tomcat and Tradesoap benchmarks, to ease the engineering requirements on our experimental setup. These benchmarks perform various kinds of I/O, spawn webservers or other processes and are generally less ameanable to profiling ${ }^{5}$.

This experiment uses as baseline not a plain static analysis (as in the previous section) but an analysis enhanced with dynamic reflection information, produced by the state-of-the-art Tamiflex tool. This is a key comparison for HeapDL. Our claim has been that heap snapshots are an excellent way to compensate for the unsoundness of static analysis, in a more complete way than merely recording specific program actions (such as reflection calls).

The experiment is conducted with the heap enricher enabled, so that the full dynamic call graph is registered (using the EdgeCtx objects), however the context information is ignored since the static analysis is carried out context-insensitively. Also, the enricher for capturing all dynamically loaded classes (Section 3.1) is not enabled, to avoid clouding the results: that enricher does not affect the HeapDL performance much on the DaCapo benchmarks and we want all analyses (static+Tamiflex vs. + HeapDL) to run on the exact same bytecode.

The heap dump is taken on JVM exit. The application is instrumented by the heap-enhancing agent to persist all relevant objects allocated by the same application. There is negligible statistical variability in the Dacapo benchmarks, since the harness is deterministic in the way it exercises the application.

Figures 12 and 13 show the number of call-graph edges and heap size for the benchmarks. The two bars are for the baseline static analysis (Doop with Tamiflex input) with and without the HeapDL input. We used the "default" input size of the DaCapo benchmarks for dynamic analysis.

As can be seen, the increase in analysis metrics is substantial, typically at over- $20 \%$ more callgraph edges (median: 24\%), and even higher for the size of the static heap (median increase: $86 \%$ ). The call-graph edge increase is smaller than on the Android setting, exactly as would be expected, since the Tamiflex input addresses some of the unsoundness of the static analysis. Tamiflex is still missing many call-graph edges, however. (We note that the increase for the batik benchmark is an outlier because Tamiflex misses a key call-graph edge with the default input. Surprisingly, it observes it with the "small" input of the benchmarks. Thus, one should not consider batik to be representative in terms of soundness, although it is still informative in terms of other metrics.) Therefore, on RQ.A, the experiment appears to strongly confirm that HeapDL is effective and improves on the state of the art.

Figure 14 shows that the increase in analysis reach comes with modest increases in static analysis cost (RQ.B2). (We even see a surprising reduction, for tradebeans. We have not yet managed to explain this, but it is a repeatable effect. We speculate that it is merely due to the analysis reaching fixpoint a lot earlier due to the many initial dynamic call-graph edges.)

The extra time taken by static analysis when enhanced with HeapDL inputs is a direct effect of enhancing the coverage of the analysis. The information exposed by HeapDL makes the analysis infer more reachable code, which in turn makes the analysis run longer. Indeed, the information (e.g., all call-graph edges, not just new ones) that the static analysis receives from HeapDL is a small proportion of the extra information that the static analysis ends up inferring. This can be seen in Figure 15, which plots the dynamic call-graph edges produced by HeapDL against the (earlier-reported) call-graph edges inferred by the static analysis with and without HeapDL. As can

\footnotetext{
${ }^{4}$ We used version $1.8 \mathrm{u} 131$ of the Oracle JDK.

${ }^{5}$ http://sourceforge.net/p/dacapobench/bugs/70/
} 


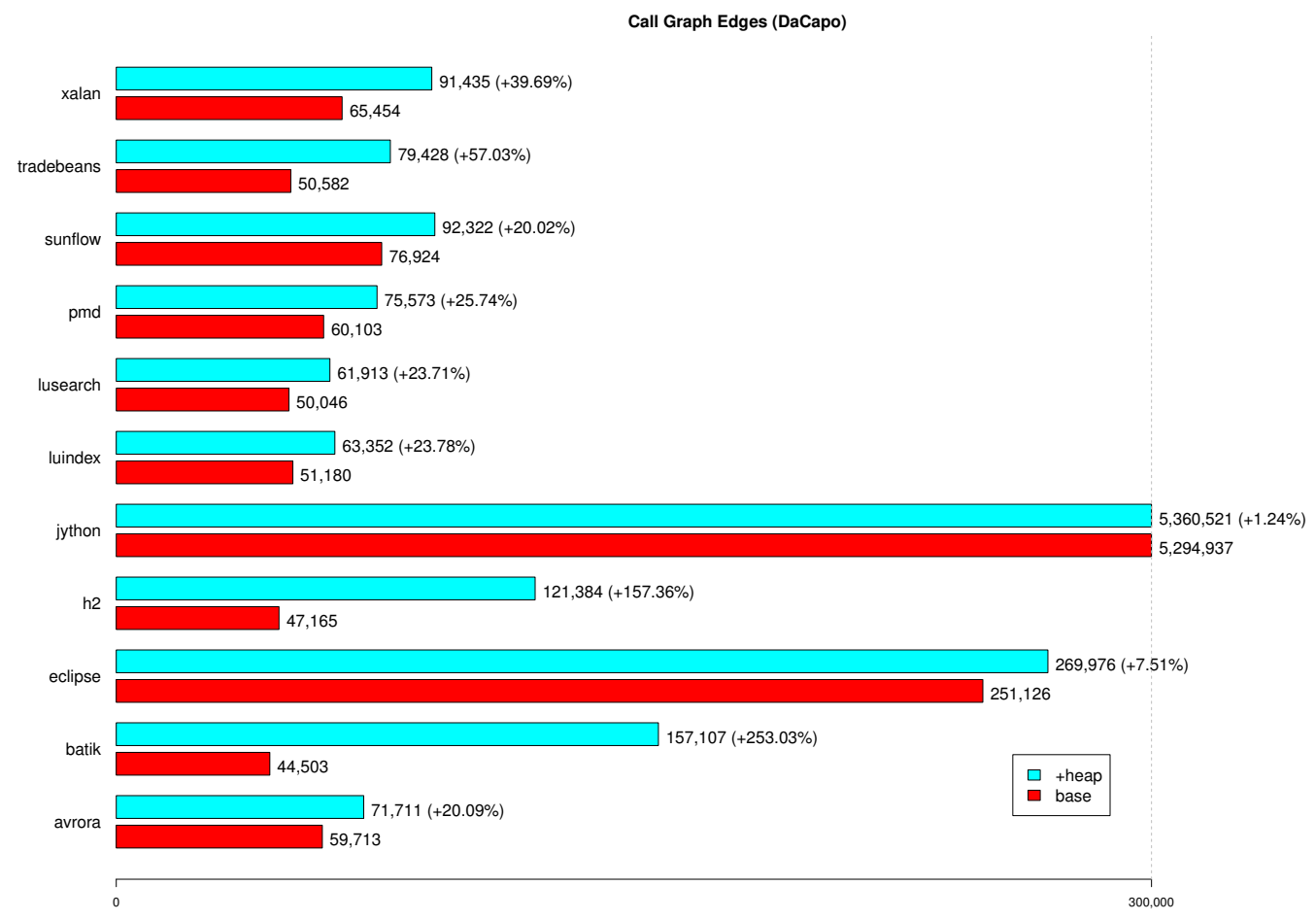

Fig. 12. Call-graph size for DaCapo benchmarks. The figure is truncated for readability.

be seen, the increase in static call-graph edges is typically $3-5 x$ of the dynamic call-graph edges that HeapDL provides.

On the other hand, the run-time cost (RQ.B1) is much higher than on the Android platform. We have found that JVM-profiling with allocation tracking on the DaCapo benchmarks incurs a 20-50x slowdown (median: 39x). This slowdown is incurred for a standard configuration of a reference profiler tool, so it is in line with overheads that programmers observe during realistic profiling tasks. Our optional heap enriching agent compounds this cost with a further 1.1-10x slowdown (median: 1.8x), for a total slowdown that can approach two-to-three orders of magnitude!

Thus, currently HeapDL pays a performance penalty on the dynamic execution in order to yield inputs for enhanced static analysis. We expect that this cost is acceptable in the majority of cases. Dynamic instrumentation often incurs high costs on high-performance platforms and the overhead does not prohibit the actual execution of realistic programs, when the stakes are as high as static (i.e., all-input) analysis coverage.

We investigated tuning options in order to minimize the profiling overhead. With a bounded depth of 6 for captured stack traces, the analysis results are nearly identical to those reported in our full experiments, yet the overhead of HPROF profiling drops to a median of 21x (instead of 39x). For future development, there are several alternative profiler implementations that can potentially yield lower overheads. These include the HPROF agent of the IBM JDK [IBM 2017], the YourKit profiler [YourKit 2017], the Java Flight Recorder [Oracle 2014a], and the Java VisualVM profiler [Oracle 2016c]. It is a strength of the approach that profiling is done externally, by third-party tools. 


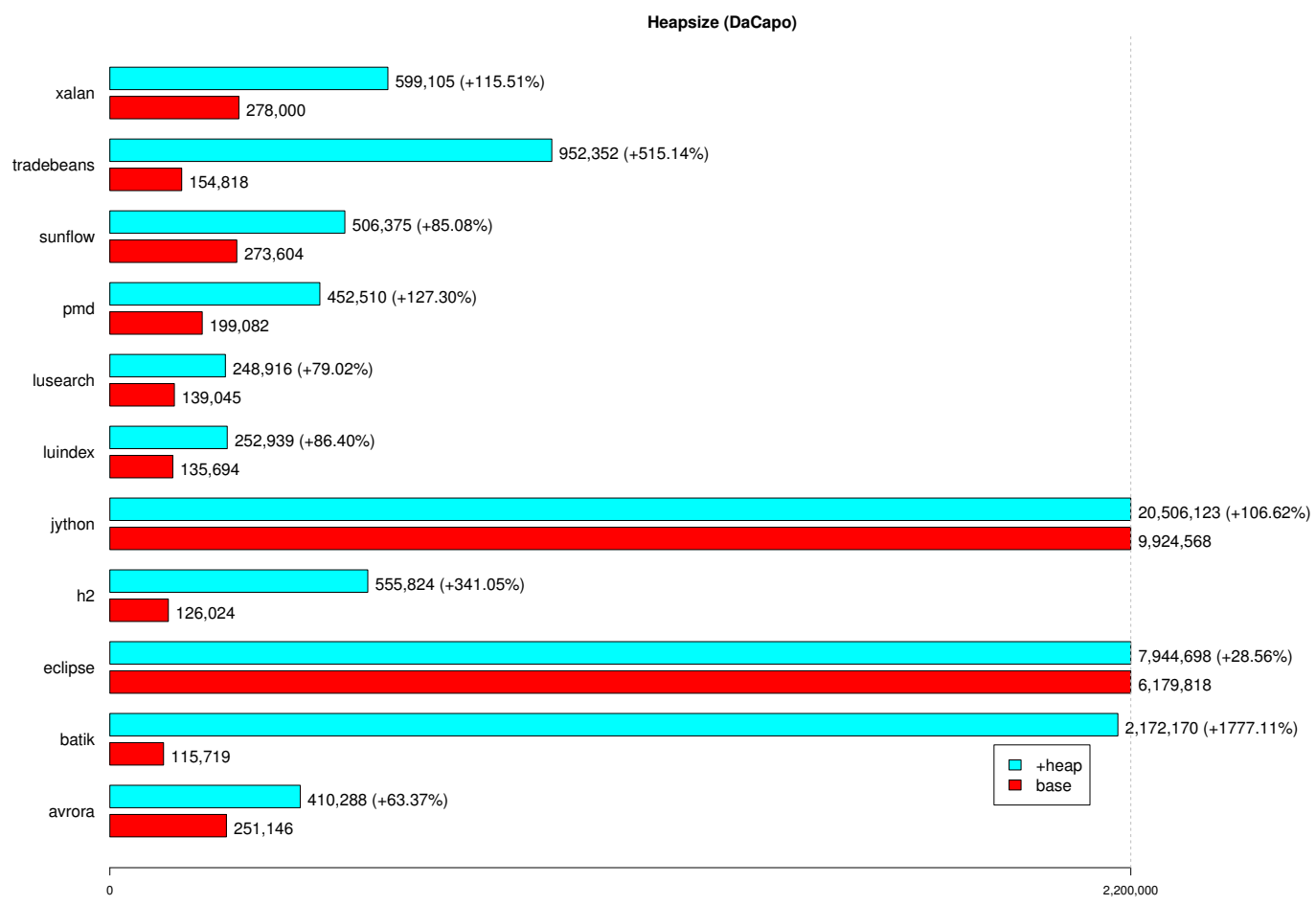

Fig. 13. Heap size for DaCapo benchmarks. The figure is truncated for readability.

\subsection{Quantifying Coverage Increase}

A highly meaningful test for mechanisms that enhance the coverage of an analysis is to measure their ability to anticipate unseen behaviors. We saw in Figure 12 that HeapDL enhances a static analysis to explore a lot more call-graph edges. But does this translate into improved coverage of behaviors that truly arise?

In order to measure the coverage increase (RQ.C) that HeapDL enables, we compare the recall of the dynamic call-graph edges for DaCapo executions under the "default" input size, when the static analysis has only seen the dynamic behavior of the "small" input size. That is, we first run the benchmarks with the "small" workload (for both the Tamiflex tool and HeapDL). This run serves to produce inputs for the static analysis, which analyzes the program and produces a static call graph. We then examine the recall of this static call graph, against the dynamic call graph arising for an execution with the "default" benchmark input. ${ }^{6}$ The setup of the experiment is otherwise identical as in the earlier DaCapo benchmark experiment, i.e., we do not enable the enricher for dynamically-loaded code, so that both Tamiflex and HeapDL operate on the same bytecode.

Figure 16 shows the results of the recall comparison. As can be seen, HeapDL results in a significant increase in call-graph edge recall: the static analysis successfully infers almost all of the dynamic call-graph edges in the "default" execution, which it has never seen. In contrast, the Tamiflex techniques alone are not enough to achieve similar coverage: more than $20 \%$ of the dynamic call-graph edges from differnt runs are missed. The median recall for the baseline (static

\footnotetext{
${ }^{6}$ We sought to perform the same experiment with the "default" vs. "large" DaCapo inputs, but this is not available for the full set of benchmarks, and others fail for the supplied "large" input, without any instrumentation.
} 


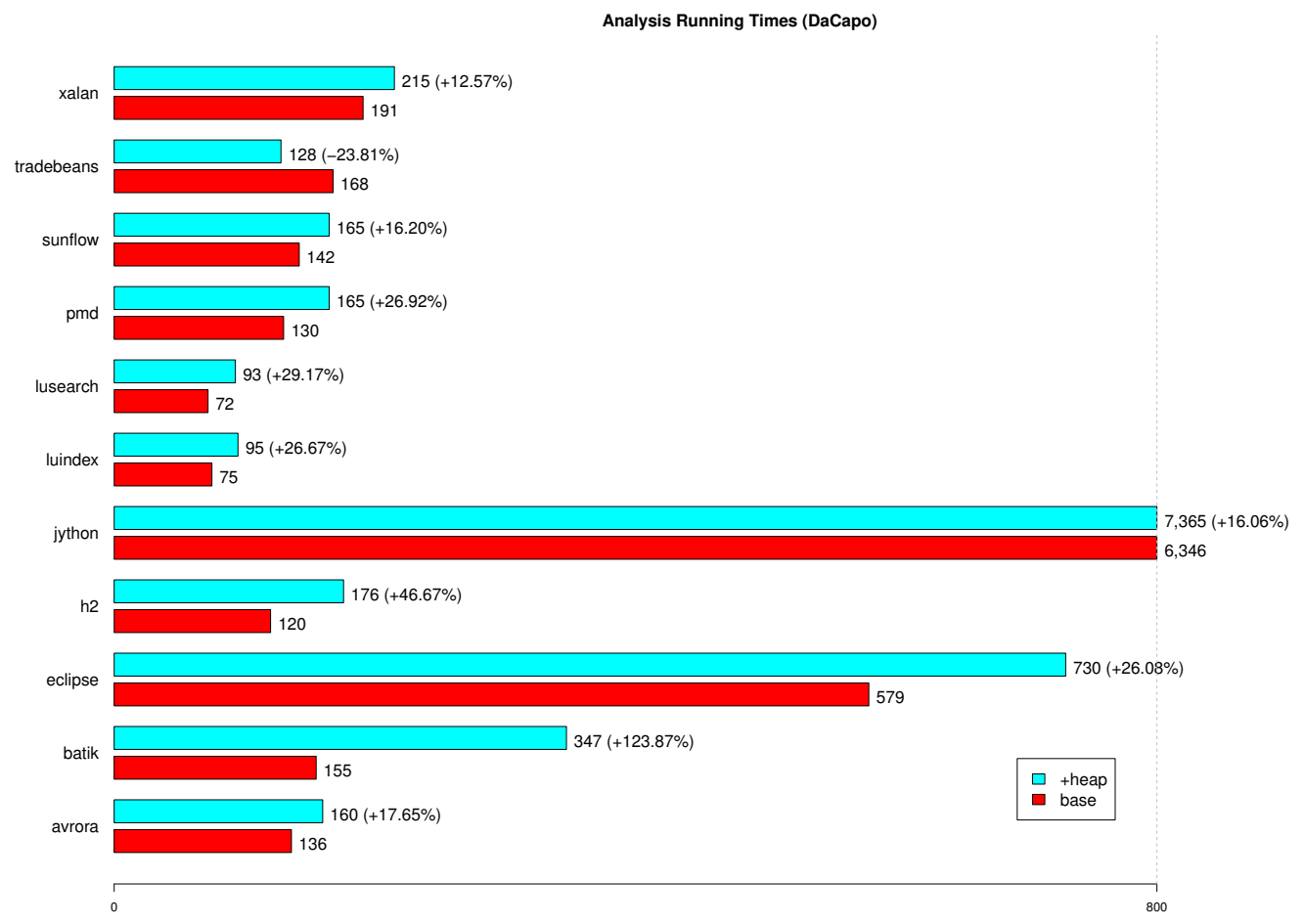

Fig. 14. Analysis times for DaCapo benchmarks. The figure is truncated for readability.

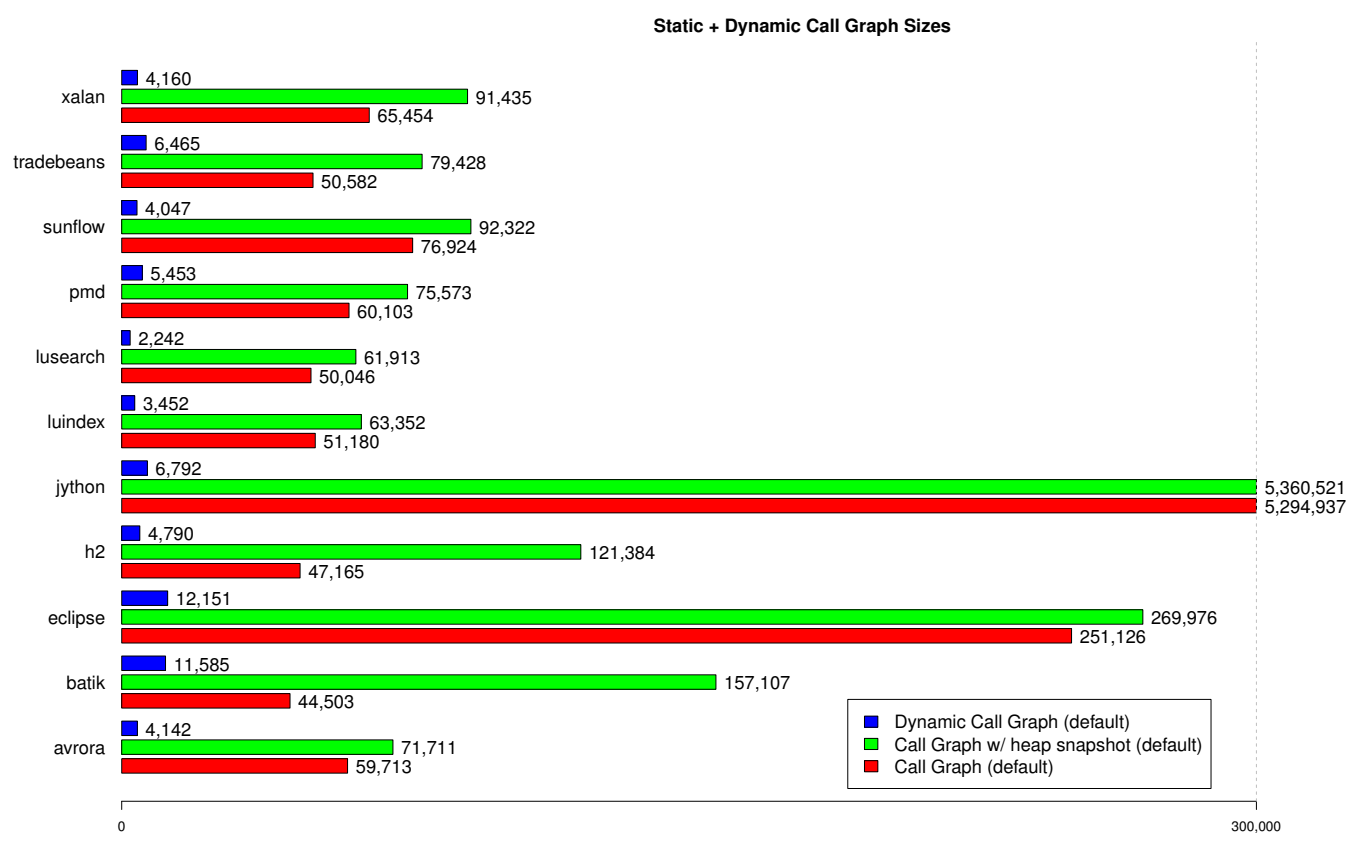

Fig. 15. Dynamic call-graph edges vs. increase in static call-graph edges. The figure is truncated for readability. 


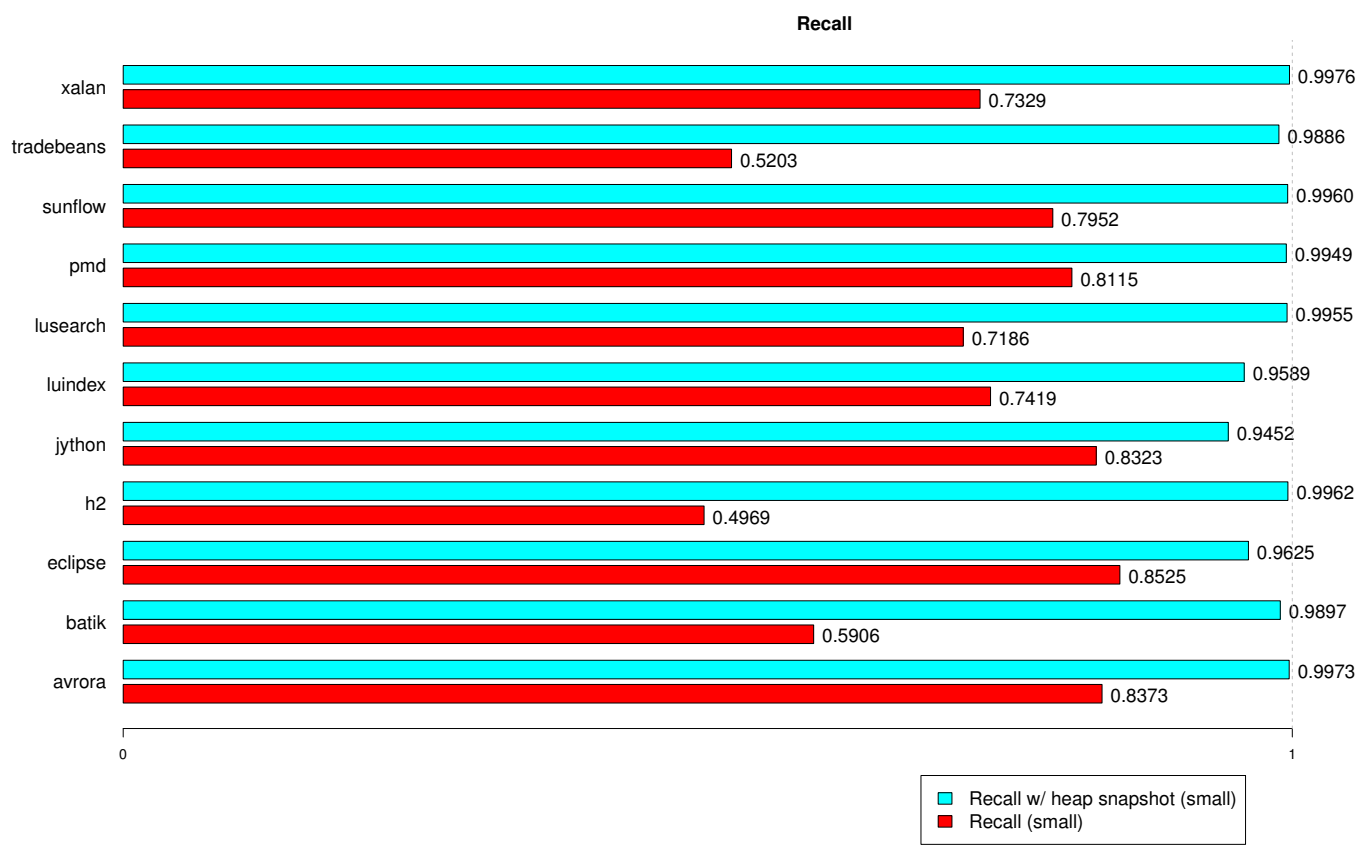

Fig. 16. Coverage/Recall for DaCapo benchmarks.

analysis + Tamiflex input) is $76.9 \%$, while it rises to $99.5 \%$ when HeapDL input is added. Thus, the experiment suggests that the answer to RQ.C is affirmative: HeapDL increases coverage of actual program behavior, compared to a state-of-the-art tool.

\subsection{Discussion}

Although we have not quantitatively classified the sources of unsoundness in our experiments, we can share qualitative insights from a manual inspection effort. Furthermore, we also show that the additional coverage gained by using HeapDL directly translates to benefits in client analyses.

5.4.1 Unsoundness in Static Analysis. Many of the dynamic edges missed by the static analysis relate to low-level code. This code, however, often translates into unsoundness when analyzing the application itself. Our earlier figures 12, 13, and 16 have 4 outliers among the DaCapo benchmarks: batik, h2, tradebeans, and xalan. All four show significant increase in the amount of application code found to be reachable by the analysis. Without HeapDL input, the static analysis often discovered less than $10 \%$ of the code of these benchmarks to be reachable. On the tradebeans benchmark, a substantial part of the edges that are missed involve typical web server functionality, e.g., encryption, security, command-line parsing, etc.

An interesting observation is that the DaCapo benchmarks are older, so they do not use the invokedynamic instruction (used, e.g., in the translation of lambda expressions). However, when analyzed in conjunction with a Java 8 library, such instructions arise: the benchmarks generate anonymous classes to be called via invokedynamic due to the automatic "SAM conversion". In new JDKs, all single-abstract-method (SAM) types see invocations of their methods transformed into invokedynamic calls [Goetz 2010; Oracle 2014b]. We have observed this arise in at least three DaCapo benchmarks. Heap snapshots successfully compensate for this semantic omission. 
In the Android setting, an example of what we gain from heap data is the discovery of many call graph edges to the graphical subsystem. Apps in Android set up their user interface using a "layout inflater", which uses reflection via external XML files to set up the GUI elements of the app. We found that such code is hostile to classic static analysis (even with reflection analysis or Tamiflex information).

5.4.2 Benefits for Client Analyses. HeapDL's effect of enhancing the call graph and reachable method coverage of static analyzers directly translates to benefits for most conceivable client analyses. For instance, for the Instagram application on Android, a static taint analyzer [Grech and Smaragdakis 2017] flags 2.6x more suspicious information flows when enhanced with HeapDL input. This is a higher-than-proportional increase in possible vulnerabilities flagged, relative to the metrics of Figures 9 and 10 on Instagram (1.6x call-graph edges, 1.7x heap size increase).

This effect is hardly surprising. At a high level, a larger coverage of reachable methods by a static analysis can easily translate into a larger number of vulnerabilities detected, e.g., vulnerabilities may lurk in the code that is not covered by the client analysis. At a more detailed level, a larger coverage of call-graph edges yields substantial increase in the behaviors covered by considering more combinations of events. For instance, in a taint analyzer, a taint source, sink, or taint transfer method is often represented as a method invocation. If the underlying analysis builds a larger, more representative call graph, the numbers of all three elements (sources, sinks, and taint transfer methods) will increase. Since an information flow consists of combinations of such events, the increase will be magnified.

\section{RELATED WORK}

The general pattern of adding dynamic analysis information to address cases that are hard for static analysis has a time-honored past, with approaches such as dynamic symbolic execution [Godefroid et al. 2005; Sen et al. 2005] and environment models in model checking. For example Mercer and Jones [2005] present a model checking approach that uses the GNU debugger to establish cycle-accurate effects of the compiled program elements under different backends and processors. What distinguishes our approach is the use of heap snapshots with allocation tracking, as well as the emphasis on the information (e.g., dynamically-loaded code, object-sensitive contexts) that is particularly valuable for a whole-program static analysis.

Li et al. [2017] show how to combine dynamic symbolic execution with subtype polymorphism in Java to resolve the targets of method invocations. Their approach improves soundness relative to plain dynamic symbolic execution but does not address the soundness issues relative to native code, heterogeneous applications, or invokedynamic.

Hirzel et al. [2004] show one of the first works that consider runtime monitoring so as to obtain information for state-of-the-art Java program analysis techniques. Concretely, they extend Andersen's pointer analysis algorithm to an online setting, which enables it to handle dynamic class loading, reflection and native code (through the disciplined JNI interface). The system observes such events and re-runs the analysis with these observations taken into account. The approach is conceptually closely related to ours, since it targets the same kinds of analyses (whole-program points-to and call-graph). However, the Hirzel et al. approach is quite different in its characteristics: it requires full control of the runtime environment; it applies only to analyses that are inexpensive enough to re-run regularly; it does not separate the dynamic information from the static analysis; it relies on capturing events and not effects (e.g., it will not intercept low-level heap updates through unsafe APIs).

Dynamic class loading with reflection is a hard problem for static analysis: Landman et al. [2017] note that there are still soundness problems in the handling of dynamic proxies or reflection, 
even with state-of-the-art techniques. Our approach partly addresses the shortcomings of these past techniques by using run-time heap information to detect behaviors that would otherwise be missed. There are also techniques that attempt to statically analyze either dynamic class loading or reflection. For example, Yoshiura and Wei [2014] do static data race detection in the presence of dynamic class loading (an analysis that is not fully automatic as it requires manual handling of loops) and Li et al. [2015] improve the static handling of reflection, but do not fully handle native methods, invokedynamic, or heterogeneous code.

Inspecting a program's state prior to static analysis is a strategy sometimes employed by hybrid static analysers for dynamically typed languages. For instance, RPython [Ancona et al. 2007] lazily inspects most of the global state, and combines dynamic and static type information to perform a flow-sensitive type inference, before generating optimized code. A similar implementation strategy is used in preemptive type checking [Grech et al. 2013]. On the other hand, the use of profiling on hard to analyse features is used in PRuby [Furr et al. 2009], particularly on Ruby's eval and other unsafe functions to infer possible side effects of these.

The Tamiflex tool [Bodden et al. 2011] (extensively discussed earlier) employs a 'Play-out' agent to $\log$ runtime reflective calls and classes loaded via custom class loaders or on-the-fly code generation. A secondary tool component called the 'Booster' then enriches the respective classes, at the point where the reflective calls are made, by inserting regular method calls that materialize the reflective calls, thus making them detectable by standard static analyzers. The Booster component also instruments runtime checks that warn the user when the analyzed program executes reflective calls that were not executed in the previous runs (and may, thus, be a source of unsoundness). The Tamiflex toolchain also provides support for inserting offline-transformed classes into a running program via a 'Play-in' agent. All such functionality would be interesting to incorporate in the HeapDL tool in the future.

Our approach is certainly not the first to recognize the high value of heap snapshots. Specifically in Java, there have been several research uses of HPROF data-e.g., most directly for different kinds of heap visualization [Aftandilian et al. 2010; Reiss 2009]. Other dynamic analyses use heap profiling data to check aliasing properties [Potanin et al. 2004], analyze synchronization performance [Hofer et al. 2015], generate software birthmarks [Chan et al. 2011], or diagnose memory leaks [Maxwell et al. 2010].

The problem of static analysis unsoundness is particularly acute for Android frameworks, since they make heavy use of reflection. Droidel [Blackshear et al. 2015] simulates some uses of reflection in Android and replaces reflective behavior with static calls to generated code (stubs); these can then be processed using off-the-shelf analysis tools for Android apps (e.g., Soot and WALA [Fink 2015]) that would otherwise miss these call-graph edges. Their approach is however not fully automated and requires manual code instrumentation for explicating reflection. In HybriDroid [Lee et al. 2016], dynamic analysis is not employed to model the effects of foreign code. Instead, the interaction between Android and JavaScript code is handled explicitly. This means that standard WebView browser component events are explicitly modeled and JavaScript code running in this is analyzed together with the application.

Zhauniarovich et al. [2015] observe that an "extensive amount of Android apps relies on dynamic code update features" and offer a combination of static and dynamic approaches for security analyses on Android. In particular, the dynamic part of their approach is using a modified Android operating system that runs the application to be analyzed. In comparison, our work differs in that it is nonintrusive. We do not need a modified version of Android to take heap snapshots (although we built a platform image from scratch to have a tightly controlled environment for benchmarks in this paper). 


\section{CONCLUSIONS}

We presented an approach to enhancing the coverage of a static analysis by employing dynamic information. Although the general pattern for such an enhancement is well-established, our techniques are interesting in their specifics. We use modern heap-snapshot and allocation-tracking technology provided by profiling APIs in mainstream platforms; we export dynamic information in a format suitable for whole-program static analysis, such as call-graph analysis and pointer analysis of a global heap; we enrich the heap for various purposes, including maintaining context information that the static analysis expects. Our approach is embodied in the HeapDL tool, which we show can achieve significant increases in analysis coverage, compared to the closest baselines.

We believe that heap snapshots are the right tool for addressing unsoundness shortcomings of static analyses. Heap snapshots offer the enormous advantage of being non-intrusive: there is no need to instrument code (except for purposes of getting optional extra information, as in heap enrichment) or, generally, to watch for specific program actions. Instead, a wealth of information on a program's behavior is readily available by observing the effects of the program on the heap and snapshot call-graphs. Such program effects can capture semantic elements of even the hardest-toanalyze code: native actions, dynamically generated code, and all sorts of unsupported functionality.

\section{ACKNOWLEDGMENTS}

We gratefully acknowledge funding by the European Research Council, grant 307334 (SPADE). In addition, the research work disclosed is partially funded by the REACH HIGH Scholars Program Post-Doctoral Grants. The grant is part-financed by the European Union, Operational Program II, Cohesion Policy 2014-2020 (Investing in human capital to create more opportunities and promote the wellbeing of society - European Social Fund).

\section{REFERENCES}

Edward E. Aftandilian, Sean Kelley, Connor Gramazio, Nathan Ricci, Sara L. Su, and Samuel Z. Guyer. 2010. Heapviz: Interactive Heap Visualization for Program Understanding and Debugging. In Proceedings of the 5th International Symposium on Software Visualization (SOFTVIS '10). ACM, New York, NY, USA, 53-62. DOI : http://dx.doi.org/10.1145/ 1879211.1879222

Davide Ancona, Massimo Ancona, Antonio Cuni, and Nicholas D. Matsakis. 2007. RPython: A Step Towards Reconciling Dynamically and Statically Typed OO Languages. In Proceedings of the 2007 Symposium on Dynamic Languages (DLS '07) ACM, New York, NY, USA, 53-64. DOI : http://dx.doi.org/10.1145/1297081.1297091

Molham Aref, Balder ten Cate, Todd J. Green, Benny Kimelfeld, Dan Olteanu, Emir Pasalic, Todd L. Veldhuizen, and Geoffrey Washburn. 2015. Design and Implementation of the LogicBlox System. In Proceedings of the 2015 ACM SIGMOD International Conference on Management of Data (SIGMOD '15). ACM, New York, NY, USA, 1371-1382. DOI : http://dx.doi.org/10.1145/2723372.2742796

Steven Arzt, Siegfried Rasthofer, Christian Fritz, Eric Bodden, Alexandre Bartel, Jacques Klein, Yves Le Traon, Damien Octeau, and Patrick McDaniel. 2014. FlowDroid: Precise Context, Flow, Field, Object-sensitive and Lifecycle-aware Taint Analysis for Android Apps. In Proceedings of the 35th ACM SIGPLAN Conference on Programming Language Design and Implementation (PLDI '14). ACM, New York, NY, USA, 259-269. DOI : http://dx.doi.org/10.1145/2594291.2594299

Stephen M. Blackburn, Robin Garner, Chris Hoffmann, Asjad M. Khan, Kathryn S. McKinley, Rotem Bentzur, Amer Diwan, Daniel Feinberg, Daniel Frampton, Samuel Z. Guyer, Martin Hirzel, Antony L. Hosking, Maria Jump, Han Bok Lee, J. Eliot B. Moss, Aashish Phansalkar, Darko Stefanovic, Thomas VanDrunen, Daniel von Dincklage, and Ben Wiedermann 2006. The DaCapo benchmarks: Java benchmarking development and analysis. In Proceedings of the 21st Annual ACM SIGPLAN Conference on Object Oriented Programming, Systems, Languages, and Applications (OOPSLA '06). ACM, New York, NY, USA, 169-190. DOI : http://dx.doi.org/10.1145/1167473.1167488

Sam Blackshear, Alexandra Gendreau, and Bor-Yuh Evan Chang. 2015. Droidel: A General Approach to Android Framework Modeling. In Proceedings of the 4th ACM SIGPLAN International Workshop on State Of the Art in Program Analysis (SOAP 2015). ACM, New York, NY, USA, 19-25. DOI : http://dx.doi.org/10.1145/2771284.2771288

Eric Bodden, Andreas Sewe, Jan Sinschek, Hela Oueslati, and Mira Mezini. 2011. Taming reflection: Aiding static analysis in the presence of reflection and custom class loaders. In Proceedings of the 33rd International Conference on Software Engineering (ICSE '11). ACM, New York, NY, USA, 241-250. DOI : http://dx.doi.org/10.1145/1985793.1985827 
Martin Bravenboer and Yannis Smaragdakis. 2009. Strictly Declarative Specification of Sophisticated Points-to Analyses. In Proceedings of the 24th Annual ACM SIGPLAN Conference on Object Oriented Programming, Systems, Languages, and Applications (OOPSLA '09). ACM, New York, NY, USA, 243-261. DOI : http://dx.doi.org/10.1145/1640089.1640108

Eric Bruneton, Romain Lenglet, and Thierry Coupaye. 2002. ASM: a code manipulation tool to implement adaptable systems. Adaptable and extensible component systems 30, 19 (2002).

Patrick P. F. Chan, Lucas C. K. Hui, and S. M. Yiu. 2011. Dynamic Software Birthmark for fava Based on Heap Memory Analysis. Springer Berlin Heidelberg, Berlin, Heidelberg, 94-107. DOI : http://dx.doi.org/10.1007/978-3-642-24712-5_8

Stephen J. Fink. 2015. T.J. Watson Libraries for Analysis (WALA). http://wala.sourceforge.net. (2015).

Michael Furr, Jong-hoon (David) An, and Jeffrey S. Foster. 2009. Profile-guided Static Typing for Dynamic Scripting Languages. In Proceedings of the 24th ACM SIGPLAN Conference on Object Oriented Programming Systems Languages and Applications (OOPSLA '09). ACM, New York, NY, USA, 283-300. DOI : http://dx.doi.org/10.1145/1640089.1640110

Patrice Godefroid, Nils Klarlund, and Koushik Sen. 2005. DART: Directed Automated Random Testing. In Proceedings of the 2005 ACM SIGPLAN Conference on Programming Language Design and Implementation (PLDI '05). ACM, New York, NY, USA, 213-223. DOI : http://dx.doi.org/10.1145/1065010.1065036

Brian Goetz. 2010. One VM, Many Languages. https://gotocon.com/dl/jaoo-aarhus-2010/slides/BrianGoetz OneVMManyLanguages.pdf. (2010).

Brian Goetz. 2016. Project Valhalla Update. http://www.oracle.com/technetwork/java/jvmls2016-goetz-3126134.pdf. (August 2016). JVM Language Summit.

Neville Grech, Julian Rathke, and Bernd Fischer. 2013. Preemptive Type Checking in Dynamically Typed Languages. In Proceedings of the 10th International Colloquium on Theoretical Aspects of Computing (Lecture Notes in Computer Science), Vol. 8049. Springer, 195-212. DOI : http://dx.doi.org/10.1007/978-3-642-39718-9_12

Neville Grech and Yannis Smaragdakis. 2017. P/Taint: Unified Points-to and Taint Analysis. Proceedings of the ACM on Programming Languages 1, OOPSLA (October 2017). DOI : http://dx.doi.org/10.1145/3133926

Martin Hirzel, Daniel Von Dincklage, Amer Diwan, and Michael Hind. 2007. Fast Online Pointer Analysis. ACM Transactions on Programming Languages and Systems 29, 2, Article 11 (April 2007), 55 pages. DOI: http://dx.doi.org/10.1145/1216374. 1216379

Martin Hirzel, Amer Diwan, and Michael Hind. 2004. Pointer Analysis in the Presence of Dynamic Class Loading. In Proceedings of the 18th European Conference on Object-Oriented Programming (ECOOP '04). Springer Berlin Heidelberg, Berlin, Heidelberg, 96-122. DOI : http://dx.doi.org/10.1007/978-3-540-24851-4_5

Peter Hofer, David Gnedt, and Hanspeter Mössenböck. 2015. Efficient Dynamic Analysis of the Synchronization Performance of Java Applications. In Proceedings of the 13th International Workshop on Dynamic Analysis (WODA 2015). ACM, New York, NY, USA, 14-18. DOI : http://dx.doi.org/10.1145/2823363.2823367

IBM. 2017. Using the HPROF Profiler. https:/www.ibm.com/support/knowledgecenter/en/SSYKE2_8.0.0/com.ibm.java.lnx. 80.doc/diag/tools/hprof.html. (2017).

Herbert Jordan, Bernhard Scholz, and Pavle Subotić. 2016. Soufflé: On Synthesis of Program Analyzers. Springer International Publishing, Cham, 422-430. DOI : http://dx.doi.org/10.1007/978-3-319-41540-6_23

George Kastrinis and Yannis Smaragdakis. 2013. Hybrid Context-Sensitivity for Points-To Analysis. In Proceedings of the 2013 ACM SIGPLAN Conference on Programming Language Design and Implementation (PLDI '13). ACM, New York, NY, USA, 423-434. DOI : http://dx.doi.org/10.1145/2491956.2462191

Davy Landman, Alexander Serebrenik, and Jurgen J. Vinju. 2017. Challenges for Static Analysis of Java Reflection - Literature Review and Empirical Study. In Proceedings of the 39th International Conference on Software Engineering, ICSE 2017, Buenos Aires, Argentina, May 20-28, 2017.

Sungho Lee, Julian Dolby, and Sukyoung Ryu. 2016. HybriDroid: Static Analysis Framework for Android Hybrid Applications. In Proceedings of the 31st IEEE/ACM International Conference on Automated Software Engineering (ASE 2016). ACM, New York, NY, USA, 250-261. DOI : http://dx.doi.org/10.1145/2970276.2970368

Lian Li, Yi Lu, and Jingling Xue. 2017. Dynamic Symbolic Execution for Polymorphism. In Proceedings of the 26th International Conference on Compiler Construction (CC 2017). ACM, New York, NY, USA, 120-130. DOI : http://dx.doi.org/10.1145/ 3033019.3033029

Yue Li, Tian Tan, and Jingling Xue. 2015. Effective Soundness-Guided Reflection Analysis. Springer Berlin Heidelberg, Berlin, Heidelberg, 162-180. DOI : http://dx.doi.org/10.1007/978-3-662-48288-9_10

Tim Lindholm, Frank Yellin, Gilad Bracha, and Alex Buckley. 2014. The Java Virtual Machine Specification, Java SE 8 Edition. (2014).

Benjamin Livshits, Manu Sridharan, Yannis Smaragdakis, Ondřej Lhoták, J. Nelson Amaral, Bor-Yuh Evan Chang, Samuel Z. Guyer, Uday P. Khedker, Anders Møller, and Dimitrios Vardoulakis. 2015. In Defense of Soundiness: A Manifesto. Commun. ACM 58, 2 (Jan. 2015), 44-46. DOI : http://dx.doi.org/10.1145/2644805

Evan K. Maxwell, Godmar Back, and Naren Ramakrishnan. 2010. Diagnosing Memory Leaks Using Graph Mining on Heap Dumps. In Proceedings of the 16th ACM SIGKDD International Conference on Knowledge Discovery and Data Mining (KDD 
'10). ACM, New York, NY, USA, 115-124. DOI : http://dx.doi.org/10.1145/1835804.1835822

Eric Mercer and Michael Jones. 2005. Model Checking Machine Code with the GNU Debugger. In Proceedings of the 12th International Conference on Model Checking Software (SPIN’05). Springer-Verlag, Berlin, Heidelberg, 251-265. DOI : http://dx.doi.org/10.1007/11537328_20

Ana Milanova, Atanas Rountev, and Barbara G. Ryder. 2005. Parameterized object sensitivity for points-to analysis for Java ACM Transactions on Software Engineering and Methodology 14, 1 (2005), 1-41. DOI : http://dx.doi.org/10.1145/1044834. 1044835

Oracle. 2014a. Java Platform, Standard Edition Java Flight Recorder Runtime Guide. https://docs.oracle.com/javacomponents/ jmc-5-4/jfr-runtime-guide/about.htm. (2014).

Oracle. 2014b. JSR 335: Lambda Expressions for the Java ${ }^{\text {TM }}$ Programming Language. (2014). https://jcp.org/en/jsr/detail?id=335

Oracle. 2016a. HPROF: A Heap/CPU Profiling Tool. http://docs.oracle.com/javase/8/docs/technotes/samples/hprof.html (2016).

Oracle. 2016b. jhat. (2016). https://docs.oracle.com/javase/8/docs/technotes/tools/unix/jhat.html

Oracle. 2016c. VisualVM: Home. https://visualvm.github.io/. (2016).

Oracle. 2017. JEP 280: Indify String Concatenation. (2017). http://openjdk.java.net/jeps/280

Alex Potanin, James Noble, and Robert Biddle. 2004. Checking Ownership and Confinement: Research Articles. Concurrency and Computation: Practice \& Experience - Formal Techniques for fava-like Programs 16, 7 (June 2004), 671-687. DOI : http://dx.doi.org/10.1002/cpe.v16:7

S. P. Reiss. 2009. Visualizing the Java heap to detect memory problems. In 2009 5th IEEE International Workshop on Visualizing Software for Understanding and Analysis. 73-80. DOI : http://dx.doi.org/10.1109/VISSOF.2009.5336418

John R. Rose. 2009. Bytecodes Meet Combinators: Invokedynamic on the JVM. In Proceedings of the Third Workshop on Virtual Machines and Intermediate Languages (VMIL '09). ACM, New York, NY, USA, Article 2, 11 pages. DOI: http://dx.doi.org/10.1145/1711506.1711508

Koushik Sen, Darko Marinov, and Gul Agha. 2005. CUTE: A Concolic Unit Testing Engine for C. In Proceedings of the 10th European Software Engineering Conference Held fointly with 13th ACM SIGSOFT International Symposium on Foundations of Software Engineering (ESEC/FSE-13). ACM, New York, NY, USA, 263-272. DOI : http://dx.doi.org/10.1145/1081706.1081750

Micha Sharir and Amir Pnueli. 1981. Two Approaches to Interprocedural Data Flow Analysis. In Program flow analysis: theory and applications, Steven S. Muchnick and Neil D. Jones (Eds.). Prentice-Hall, Inc., Englewood Cliffs, NJ, Chapter 7 , 189-233.

Yannis Smaragdakis, Martin Bravenboer, and Ondřej Lhoták. 2011. Pick Your Contexts Well: Understanding Object-Sensitivity. In Proceedings of the 38th ACM SIGPLAN-SIGACT Symposium on Principles of Programming Languages (POPL '11). ACM, New York, NY, USA, 17-30. DOI : http://dx.doi.org/10.1145/1926385.1926390

Raja Vallée-Rai, Phong Co, Etienne Gagnon, Laurie J. Hendren, Patrick Lam, and Vijay Sundaresan. 1999. Soot - a Java bytecode optimization framework. In Proceedings of the 1999 Conference of the Centre for Advanced Studies on Collaborative research (CASCON '99). IBM Press, 125-135.

Noriaki Yoshiura and Wei Wei. 2014. Static Data Race Detection for Java Programs with Dynamic Class Loading. Springer International Publishing, Cham, 161-173. DOI : http://dx.doi.org/10.1007/978-3-319-11692-1_14

YourKit. 2017. https://www.yourkit.com/. (2017).

Yury Zhauniarovich, Maqsood Ahmad, Olga Gadyatskaya, Bruno Crispo, and Fabio Massacci. 2015. StaDynA: Addressing the Problem of Dynamic Code Updates in the Security Analysis of Android Applications. In Proceedings of the 5th ACM Conference on Data and Application Security and Privacy (CODASPY '15). ACM, New York, NY, USA, 37-48. DOI : http://dx.doi.org/10.1145/2699026.2699105

Proceedings of the ACM on Programming Languages, Vol. 1, No. OOPSLA, Article 68. Publication date: October 2017. 